\title{
Higher loop nonplanar anomalous dimensions from symmetry
}

\section{Robert de Mello Koch, Stuart Graham and Ilies Messamah}

National Institute for Theoretical Physics, School of Physics and Centre for Theoretical Physics, University of Witwatersrand, Wits, 2050, South Africa

E-mail: robert@neo.phys.wits.ac.za, trautsgraham@gmail.com,

Ilies.Messamah@wits.ac.za

ABSTRACT: In this article we study the action of the one loop dilatation operator on operators with a classical dimension of order $N$. These operators belong to the $s u(2)$ sector and are constructed using two complex fields $Y$ and $Z$. For these operators nonplanar diagrams contribute already at the leading order in $N$ and the planar and large $N$ limits are distinct. The action of the one loop and the two loop dilatation operator reduces to a set of decoupled oscillators and factorizes into an action on the $Z$ fields and an action on the $Y$ fields. Direct computation has shown that the action on the $Y$ fields is the same at one and two loops. In this article, using the $s u(2)$ symmetry algebra as well as structural features of field theory, we give compelling evidence that the factor in the dilatation operator that acts on the $Y$ s is given by the one loop expression, at any loop order.

KEYwords: Supersymmetric gauge theory, AdS-CFT Correspondence, 1/N Expansion

ARXIV EPRINT: 1312.6227 


\section{Contents}

1 Summary and conclusions 1

2 Action of $s u(2)$ elements on restricted Schur polynomials 3

3 Recursion relations and one loop dilatation operator $\quad 11$

4 Continuum limit $\quad 18$

5 Differential equations and higher loop anomalous dimensions 21

5.1 One loop 25

5.2 General discussion 31

A The relation between $f_{c, d}^{(n, m)}\left(x_{j}, x_{j^{3}}\right)$ and $f_{c, d}^{(n-1, m+1)}\left(x_{j}, x_{j^{3}}\right)$

\section{Summary and conclusions}

The study of the spectrum of planar anomalous dimensions in $\mathcal{N}=4$ super Yang-Mills theory, motivated largely by the AdS/CFT correspondence [1-3], has been extremely fruitful. A key ingredient in this success has been the discovery that the planar dilatation operator can be identified with the Hamiltonian of an integrable spin chain [4-6]. It is interesting to ask if integrability persists beyond the planar limit. There has recently been some progress in this direction in [7-10] which argues the action of the one loop dilatation operator on operators [11-15] with a classical dimension of order $N$, reduces to a set of decoupled oscillators. The correlation functions of these operators receive contributions from non-planar diagrams even at the leading order in a large $N$ expansion [16]. This makes the study of correlators of these operators challenging. Techniques employing group representation theory have been very effective for this problem [12-15, 17-25]. There are two natural ways in which this work can be extended: one can try to study the action of the complete one loop dilatation operator or one can try to extend known results to higher loops. We will pursue the second goal in this article, considering the action of the dilatation operator at higher loops in the $s u(2)$ sector. This sector is particularly simple, and so it provides the ideal setting in which to develop the necessary methods.

The operators we study are built using the complex adjoint scalars $Z$ and $Y$. We use $n Z$ fields and $m Y$ fields. We will keep $n$ to be order $N$ and $m$ to be order $\sqrt{N}$. The operators that we study, the restricted Schur polynomials, $O_{R,(r, s) \vec{\mu}}$ are labeled by three Young diagrams $R, r$ and $s$ as well as a multiplicity label $\vec{\mu}$. Young diagram $r$ contains $n$ boxes and the reader is encouraged to think of $r$ as a symmetric group representation that organizes the $Z$ fields. Similarly, $s$ is a Young diagram with $m$ boxes, and it together 
with the multiplicity label $\vec{\mu}$ organizes the $Y$ fields. Roughly speaking, the Young diagram $R$ (which has $m+n$ boxes) tells us how the two sets of fields are combined. ${ }^{1}$ The pair of Young diagrams $(r, s)$ label an irreducible representation of the $S_{n} \times S_{m}$ subgroup of $S_{n+m}$. Upon restricting to the $S_{n} \times S_{m}$ subgroup, the $S_{n+m}$ irreducible representation $R$ can subduce many copies of $(r, s)$. The multiplicity labels $\vec{\mu}$ keep track of which copy of $(r, s)$ we are using. See equation (2.5) below for the definition of the restricted Schur polynomial. We study operators labeled by Young diagrams $r$ with two long rows. These operators are dual to a system of two giant gravitons (built from the $Z \mathrm{~s}$ ) dressed by open strings (given by the $Y \mathrm{~s}$ ).

The one loop and two loop answers for the spectrum of anomalous dimensions show an interesting pattern. The action of the dilatation operator at one loop and at two loops factorizes into a piece that acts only on the $r$ label - i.e. on the $Z$ fields and a piece that acts only on the $s$ and $\vec{\mu}$ labels, i.e. on the $Y$ fields. Further at one loop and at two loop the factor that acts on the $Y$ fields is identical [26]. This prompts a very natural question: does this persist at higher loops? In this article we will argue that it does.

A brute force field theoretic approach to this problem seems hopeless. Here however, we can take some guidance from progress made in the planar sector of the theory [27]. Indeed, working in the $s u(2 \mid 3)$ sector of theory and using the symmetry algebra as well as structural features from field theory, a great deal of information was obtained about higher loop corrections to the dilatation operator [27]. In the $s u(2)$ sector that we study, we have operators $\vec{J}$ that generate an $\mathrm{SU}(2)$ subgroup of the full $\mathrm{SU}(4) \mathcal{R}$ symmetry enjoyed by the theory. The $\vec{J}$ rotate the $Y$ and $Z$ fields amongst each other. Since their eigenvalues are fixed by the $s u(2)$ algebra, we know that these generators do not receive quantum corrections. One of our results is a concrete expression for the action of these generators, in the large $N$ limit, on restricted Schur polynomials. This is described in section 2 . In contrast to the operators $\vec{J}$ the dilatation operator does receive quantum corrections. Since the operators $\vec{J}$ commute with the dilatation operator, we do have some information about higher loop corrections. Using this algebra, together with the large $N$ limit and the constraints that follow from the fact that the dilatation operator is constructed by summing Feynman diagrams, we will give compelling evidence that the factor in the dilatation operator that acts on the $Y \mathrm{~s}$ is given by the one loop expression at any loop order. Concretely, the algebra $[\vec{J}, D]=0$ implies a set of recursion relations, hermitticity of the dilatation operator equates certain matrix elements of $D$ and the fact that we work at large $N$ implies that we can neglect changes in Young diagram $r$ and further that the relation between $R$ and $r$ is preserved by $D .{ }^{2}$ The derivation of these recursion relations and the structure of the dilatation operator and a demonstration that they determine the one loop dilatation operator is carried out in section 3 . This analysis is most easily extended to higher loops by employing a continuum limit. The structure of this

\footnotetext{
${ }^{1}$ Instead of saying that $R$ is a Young diagram with $m+n$ we will use the standard notation $R \vdash m+n$. Similarly, $r \vdash n$ and $s \vdash m$.

${ }^{2} r$ is obtained by removing boxes from $R$. When we say that the relation between $R$ and $r$ is preserved by $D$, we mean that $D$ will only mix operators that are obtained by pulling the same number of boxes from each row of the big Young diagram $R$ to obtain $r$.
} 
continuum limit is developed in section 4 . In section 5 we demonstrate that the recursion relations derived in section 3 are replaced by partial differential equations. These partial differential equations describe all higher loops corrections to the dilatation operator. As we explain in section 5 , they can be solved rather completely.

The fact that the factor in the dilatation operator that acts on the $Y \mathrm{~s}$ is given by the one loop expression at any loop order is not completely unexpected. Indeed, the diagonalization of this factor, achieved in general in [10], gives the set of states that is consistent with the Gauss Law constraints on a compact giant graviton world volume [11]. We expect these constraints to be satisfied at any order in the loop expansion, because the Gauss Law is an exact statement.

For simplicity we have restricted ourselves to the sector of the theory that is dual to a system of two giant gravitons. It would be straight forward but rather tedious to extend this to systems of more than two giant gravitons. A much more interesting generalization is to go beyond the $s u(2)$ sector, because symmetry is not very constraining in the $s u(2)$ sector. This follows because the dilatation operator is abelian and not part of a bigger algebra. Restricted Schur polynomials for the $s u(2 \mid 3)$ sector have been derived in [28] and the use of symmetry in this sector would represent a very interesting generalization.

Another problem that should be tackled is to determine the factor in the dilatation operator that acts on the $Z$ label. Understanding this factor, together with the results of this paper, would allow a determination of the exact large $N$ anomalous dimensions. This is not as unexpected as one might expect. Indeed, the operators we study are dual to giant gravitons. One expects the local relativistic invariant world volume theory dynamics to emerge from the sector of the theory we are considering. This picture suggests a relatively simple expression for the anomalous dimensions, determined by relativistic dispersion relations. The simplicity we find in this paper is the first signal that this expectation is correct. For closely related discussions see $[29,30]$.

\section{Action of $s u(2)$ elements on restricted Schur polynomials}

In this section our goal is to compute the action of the generators $J_{ \pm}$and $J_{3}$ on restricted Schur polynomials. We will freely make use of the results obtained in [8] in this section. Recall that in terms of the complex coordinates $z$ and $y$, we can realize the $\operatorname{su}(2)$ algebra as follows

$$
J_{+}=y \frac{\partial}{\partial z}, \quad J_{-}=z \frac{\partial}{\partial y}, \quad J_{3}=y \frac{\partial}{\partial y}-z \frac{\partial}{\partial z} .
$$

This follows because $\mathrm{SU}(2)$ rotates the complex coordinates into each other. These generators close the usual algebra

$$
\left[J_{+}, J_{-}\right]=J_{3}, \quad\left[J_{3}, J_{ \pm}\right]= \pm 2 J_{ \pm} .
$$

When acting on the restricted Schur polynomials the generators are

$$
J_{+}=\operatorname{Tr}\left(Y \frac{d}{d Z}\right), \quad J_{-}=\operatorname{Tr}\left(Z \frac{d}{d Y}\right), \quad J_{3}=\operatorname{Tr}\left(Y \frac{d}{d Y}\right)-\operatorname{Tr}\left(Z \frac{d}{d Z}\right) .
$$


This follows because the $\mathrm{SU}(2) \mathcal{R}$-symmetry rotates the matrices $Z$ and $Y$ into each other. In what follows we will make use of the identity [31]

$$
\operatorname{Tr}\left(\sigma Y^{\otimes m} \otimes Z^{\otimes n}\right)=\sum_{T, t, u, \vec{\nu}} \frac{d_{T} n ! m !}{d_{t} d_{u}(n+m) !} \chi_{T,(t, u) \vec{\nu}^{*}}\left(\sigma^{-1}\right) \chi_{T,(t, u) \vec{\nu}}(Z, Y)
$$

where if $\vec{\nu}=\left(\nu_{1}, \nu_{2}\right)$ then $\vec{\nu}^{*}=\left(\nu_{2}, \nu_{1}\right)$. With a suitable choice of $\sigma$, the right hand side above gives any desired multitrace operator. Thus, the above equation explains how to write an arbitrary multitrace operator as a linear combination of restricted Schur polynomials. The sum above runs over all Young diagrams $T \vdash m+n, t \vdash n$ and $u \vdash m$ as well as over the multplicity labels $\vec{\nu} . d_{T}$ denotes the dimension of the irreducible representation $T$ of $S_{n+m}$. Similarly, $d_{t}$ denotes the dimension of irreducible representation $t$ of $S_{n}$ and $d_{u}$ the dimension of irreducible representation $u$ of $S_{m}$. Finally, $\chi_{T,(t, u) \vec{\nu}^{*}}\left(\sigma^{-1}\right)$ is the restricted character obtained by tracing $\Gamma_{R}\left(\sigma^{-1}\right)$ over the $(t, u)$ subspace. The multiplicity index $\vec{\nu}^{*}=\left(\nu_{2}, \nu_{1}\right)$ tells us to trace the row index over the $\nu_{2}$ copy of $(r, s)$ and the column index over the $\nu_{1}$ copy.

Consider a system of $g$ giant gravitons, i.e. the Young diagrams labeling the restricted Schur polynomials have a total of $g$ rows. Our operators are built using $n Z$ fields and $m$ $Y$ fields. Our operators are $(r \vdash n$ and $s \vdash m)$

$$
\chi_{R,(r, s) \vec{\mu}}(Z, Y)=\frac{1}{n ! m !} \sum_{\sigma \in S_{n+m}} \operatorname{Tr}_{(r, s) \vec{\mu}}\left(\Gamma^{R}(\sigma)\right) \operatorname{Tr}\left(\sigma Y^{\otimes m} \otimes Z^{\otimes n}\right) .
$$

In the above, $\vec{\mu}$ is a multiplicity label. The restricted trace can be written in terms of an intertwining map $P_{R,(r, s) \vec{\mu}}$ as

$$
\operatorname{Tr}_{(r, s) \vec{\mu}}(\cdots)=\operatorname{Tr}\left(P_{R,(r, s) \vec{\mu}} \cdots\right)
$$

which factorizes as $[8]$

$$
P_{R,(r, s) \vec{\mu}}=p_{s \vec{\mu}} \otimes \mathbf{1}_{r}
$$

It is possible to compute $P_{R,(r, s) \vec{\mu}}$ explicitely for restricted Schur polynomials that are labeled by Young diagrams $R$ with long rows and well separated corners [8]. We call this the displaced corners approximation. Recall that $n \gg m$ and that $R$ has $g$ long rows. We hold $g$ fixed and order 1 as we take $N \rightarrow \infty$. In this limit the difference in the lengths of the corresponding rows of $R$ and $r$ can be neglected. Let $V_{g}$ be a $g$ dimensional vector space. In the construction of the projectors we removed $m$ boxes from $R$ to produce $r$ with each box represented by a vector in $V_{g}$. The matrix $E_{i j}$ acting in $V_{g}$ is a $g \times g$ matrix with a 1 in the $i^{\text {th }}$ row and $j^{\text {th }}$ column, and zeros elsewhere. The space $V_{g}^{\otimes k}$ obtained by tensoring $k$ copies of $V_{g}$ will also play a role in what follows. The matrix $E_{i j}^{(a)}$ acts as $E_{i j}$ on the $a^{\text {th }}$ copy of $V_{g}$ in $V_{g}^{\otimes k}$ and as the identity on all other copies. In the displaced corners approximation the multiplicity label is a pair of Gelfand-Tsetlin patterns. Both the space $V_{g}^{\otimes k}$ as well as the $E_{i j}^{(a)}$ will play an important role in the computations that follow. For more details and background see [8]. Consider the action of $J_{-}$ 


$$
\begin{aligned}
& J_{-} \chi_{R,(r, s) \vec{\mu}}(Z, Y)=\operatorname{Tr}\left(Z \frac{d}{d Y}\right) \chi_{R,(r, s) \vec{\mu}}(Z, Y) \\
& =\frac{m}{n ! m !} \sum_{\sigma \in S_{n+m}} \operatorname{Tr}_{(r, s) \vec{\mu}}\left(\Gamma^{R}(\sigma)\right) \operatorname{Tr}\left(\sigma Y^{\otimes m-1} \otimes Z^{\otimes n+1}\right) \\
& =\frac{m}{n ! m !} \sum_{\sigma \in S_{n+m}} \operatorname{Tr}_{(r, s) \vec{\mu}}\left(\Gamma^{R}(\sigma)\right) \sum_{T,\left(t^{+}, u^{-}\right) \vec{\nu}} \frac{d_{T}(n+1) !(m-1) !}{d_{t^{+}} d_{u^{-}}(n+m) !} \chi_{T,\left(t^{+}, u^{-}\right) \vec{\nu}^{*}}\left(\sigma^{-1}\right) \chi_{T,\left(t^{+}, u^{-}\right) \vec{\nu}}(Z, Y) \\
& =\sum_{T,\left(t^{+}, u^{-}\right) \vec{\nu}} \frac{d_{T}(n+1)}{d_{t^{+}} d_{u^{-}}(n+m) !} \frac{(n+m) !}{d_{T}} \delta_{R T} \operatorname{Tr}_{R \oplus T}\left(P_{R,(r, s) \vec{\mu}} P_{\left.T,\left(t^{+}, u^{-}\right) \vec{\nu}^{*}\right) \chi_{T,\left(t^{+}, u^{-}\right) \vec{\nu}}(Z, Y)}=\sum_{\left(t^{+}, u^{-}\right) \vec{\nu}} \frac{n+1}{d_{t^{+}} d_{u^{-}}} \operatorname{Tr}_{R}\left(P_{R,(r, s) \vec{\mu}} P_{R,\left(t^{+}, u^{-}\right) \vec{\nu}^{*}}\right) \chi_{R,\left(t^{+}, u^{-}\right) \vec{\nu}}(Z, Y) .\right.
\end{aligned}
$$

In the above expression $t^{+}$is a Young diagram with $n+1$ boxes, $t^{+} \vdash n+1$. The + superscript indicates that a box has been added to $t$. Similarly $u^{-} \vdash m-1$ with the superscript indicating that a box has been removed from $u$. Let us now discuss how to perform the trace in the above expression. Using the factorized form of the intertwining map in (2.7), we have [8]

$$
\operatorname{Tr}_{R}\left(P_{R,(r, s) \vec{\mu}} P_{R,\left(t^{+}, u^{-}\right) \vec{\nu}^{*}}\right)=\operatorname{Tr}_{R}\left(p_{s \vec{\mu}} \otimes \mathbf{1}_{r} \cdot p_{u^{-} \vec{\nu}^{*}} \otimes \mathbf{1}_{t^{+}}\right) .
$$

The only way that this trace can be non-zero is if it is possible for $t^{+}$to subduce $r$. Write the projector $\mathbf{1}_{t^{+}}$in terms of its action on the $\mathrm{m}^{\text {th }}$ slot and $\mathbf{1}_{r}$. As an example to illustrate the idea, consider

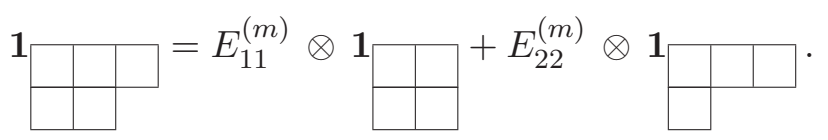

In the same way, if $t_{i}^{+\prime}=r$ we have ${ }^{3}$

$$
\mathbf{1}_{t^{+}}=E_{i i}^{(m)} \otimes \mathbf{1}_{r}+\cdots
$$

where $\cdots$ collects the terms that don't contribute to the value of the trace. Consequently, in the displaced corners approximation we find [8]

$$
\begin{aligned}
\operatorname{Tr}_{R}\left(P_{R,(r, s) \vec{\mu}} P_{R,\left(t^{+}, u^{-}\right) \vec{\nu}^{*}}\right) & =\operatorname{Tr}_{R}\left(p_{s \vec{\mu}} \otimes \mathbf{1}_{r} \cdot p_{u^{-} \vec{\nu}^{*}} \otimes \mathbf{1}_{t^{+}}\right) \\
& =\sum_{i} d_{r} \operatorname{Tr}_{V_{g} \otimes m}\left(p_{s \vec{\mu}} \cdot p_{u^{-} \vec{\nu}^{*}} \otimes E_{i i}^{(m)}\right) \delta_{t_{i}^{+\prime} r} .
\end{aligned}
$$

To proceed further, recall that the multiplicity labels $\vec{\mu}$ and $\vec{\nu}$ stand for Gelfand-Tsetlin patterns, that is, states of $\mathrm{U}(g)$. In addition, $E_{i i}=|\vec{v}(i)\rangle\langle\vec{v}(i)|$ and there is no sum on $i$. The state $|\vec{v}(i)\rangle$ is a state in the fundamental of $\mathrm{U}(g)$ - it is a $g$ dimensional vector of zeros except for the $\mathrm{i}^{\text {th }}$ entry which is a 1 . The projector $p_{s \vec{\mu}}$ is $[8]$

$$
p_{s \vec{\mu}}=\sum_{a=1}^{d_{s}}\left|M_{s}^{\mu_{1}}, a\right\rangle\left\langle M_{s}^{\mu_{2}}, a\right|
$$

\footnotetext{
${ }^{3} t_{i}^{+\prime}$ is the Young diagram obtained by dropping a box from the $\mathrm{i}^{\text {th }}$ row of $t^{+}$.
} 
where $\left|M_{s}^{\mu_{1}}, a\right\rangle$ is a state labeled by a Gelfand-Tsetlin pattern. $M_{s}^{\mu_{1}}$ is the pattern and $a$ labels states inside symmetric group irreducible representation $s$. This state is obtained by taking a suitable linear combination of tensor products of $m$ copies (one for each slot) of the fundamental representation of $\mathrm{U}(\mathrm{g})$. Rewrite this state as a linear combination of states which are each the tensor product of the fundamental representation for the $m^{\text {th }}$ slot, with a state obtained by taking the tensor product of states of the remaining $m-1$ slots $^{4}$

$$
\left|M_{s}^{\mu_{1}}, a\right\rangle=\sum_{M_{s^{\prime}, M_{F}}^{\alpha_{1}}} C_{M_{s^{\prime}}^{\alpha_{1}}, M_{F}^{l}}^{M_{1}^{\mu_{1}}}\left|M_{s^{\prime}}^{\alpha_{1}}, b\right\rangle \otimes\left|M_{F}^{l}\right\rangle .
$$

$\left|M_{F}^{l}\right\rangle$ stands for a state in the fundamental representation of $\mathrm{U}(g),\left|M_{F}^{l}\right\rangle=|\vec{v}(l)\rangle$. When $E_{i i}^{(m)}$ acts on $\left|M_{s}^{\mu_{1}}, a\right\rangle$ it will pick out the piece with $l=i$. Thus,

$$
\begin{aligned}
\operatorname{Tr}_{V_{g}^{\otimes m}}\left(p_{s \vec{\mu}} \cdot p_{u^{-} \vec{\nu}^{*}} \otimes E_{i i}^{(m)}\right) & =C_{M_{s^{\prime}}^{\alpha_{1}}, M_{F}^{i}}^{M_{F}^{\mu_{1}}} C_{M_{s^{\prime}}^{\alpha_{2}}, M_{F}^{i}}^{M_{F}^{\mu_{2}}} \operatorname{Tr}_{V_{g}^{\otimes m-1}}\left(p_{s^{\prime} \vec{\alpha}} \cdot p_{u^{-} \vec{\nu}^{*}}\right) \\
& =d_{u^{-}} C_{M_{u^{-}}^{\nu_{1}}, M_{F}^{i}}^{M_{\mu^{\prime}}} C_{M_{u^{-}}^{\nu_{2}}, M_{F}^{i}}^{M_{2}^{\mu_{2}}} .
\end{aligned}
$$

The Clebsch-Gordan coefficient can be written is in terms of bras and kets as follows

$$
C_{M_{u^{-}}^{\nu_{1}, M_{F}^{i}}}^{M_{1}^{\mu_{1}}}=\left\langle\nu_{1} \otimes \vec{v}(i) \mid \mu_{1}\right\rangle
$$

Using this notation we finally have

$$
\operatorname{Tr}_{R}\left(P_{R,(r, s) \vec{\mu}} P_{R,\left(t^{+}, u^{-}\right) \vec{\nu}^{*}}\right)=\sum_{i} d_{r} d_{u^{-}}\left\langle\mu_{2} \mid \nu_{2} \otimes \vec{v}(i)\right\rangle\left\langle\nu_{1} \otimes \vec{v}(i) \mid \mu_{1}\right\rangle \delta_{t_{i}^{+\prime} r}
$$

Thus,

$$
\begin{aligned}
J_{-} \chi_{R,(r, s) \vec{\mu}}(Z, Y) & =\sum_{\left(t^{+}, u^{-}\right) \vec{\nu}} \frac{n+1}{d_{t^{+}} d_{u^{-}}} \operatorname{Tr}_{R}\left(P_{R,(r, s) \vec{\mu}} P_{R,\left(t^{+}, u^{-}\right) \vec{\nu}^{*}}\right) \chi_{R,\left(t^{+}, u^{-}\right) \vec{\nu}} \\
& =\sum_{\left(t^{+}, u^{-}\right) \vec{\nu}} \sum_{i} \delta_{R T} \delta_{t_{i}^{+{ }^{\prime}} r} \frac{(n+1) d_{r}}{d_{t^{+}}}\left\langle\mu_{2} \mid \nu_{2} \otimes \vec{v}(i)\right\rangle\left\langle\nu_{1} \otimes \vec{v}(i) \mid \mu_{1}\right\rangle \chi_{R,\left(t^{+}, u^{-}\right) \vec{\nu}} .
\end{aligned}
$$

We want the action on normalized operators. The two point function of our operators are [15]

$$
\left\langle\chi_{R,(r, s) \vec{\mu}}(Z, Y) \chi_{T,(t, u) \vec{\nu}}^{\dagger}(Z, Y)\right\rangle=\delta_{R T} \delta_{r t} \delta_{s u} \delta_{\vec{\mu} \vec{\nu}} \frac{f_{R} \operatorname{hooks}_{R}}{\operatorname{hooks}_{r} \operatorname{hooks}_{s}} .
$$

By rescaling we can get operators with two point function equal to 1 . Denote these by $O_{R,(r, s) \vec{\mu}}(Z, Y)$. Acting on the normalized operators we have

$$
J_{-} O_{R,(r, s) \vec{\mu}}(Z, Y)=\sum_{T,\left(t^{+}, u^{-}\right) \vec{\nu}}\left(J_{-}\right)_{T,\left(t^{+}, u^{-}\right) \vec{\nu}, R,(r, s) \vec{\mu}} O_{T,\left(t^{+}, u^{-}\right) \vec{\nu}}(Z, Y)
$$

\footnotetext{
${ }^{4}$ It is useful to spell out the index structure of the next equation. The index $a$ runs over states in $S_{m}$ irreducible representation $s$. The index $b$ runs over states in irreducible representations $s^{\prime}$ subduced by $s$ when $S_{m}$ is restricted to $S_{m-1}$. We can thus put $a$ and the sets of different $b$ indices (one for every $s^{\prime}$ ) into correspondence.
} 
where

$$
\begin{aligned}
& \left(J_{-}\right)_{T,\left(t^{+}, u^{-}\right) \vec{\nu}, R,(r, s) \vec{\mu}}=\sqrt{\frac{\text { hooks }_{r} \text { hooks }_{s}}{\text { hooks }_{t^{+}} \text {hooks }_{u^{-}}}} \\
& \times \sum_{i} \delta_{R T} \delta_{t_{i}^{+\prime} r} \frac{(n+1) d_{r}}{d_{t^{+}}}\left\langle\mu_{2} \mid \nu_{2} \otimes \vec{v}(i)\right\rangle\left\langle\nu_{1} \otimes \vec{v}(i) \mid \mu_{1}\right\rangle \\
& =\sqrt{\frac{\text { hooks }_{t^{+}} \text {hooks }_{s}}{\text { hooks }_{r} \text { hooks }_{u^{-}}}} \sum_{i} \delta_{R T} \delta_{t_{i}^{+{ }_{r}}{ }_{r}}\left\langle\mu_{2} \mid \nu_{2} \otimes \vec{v}(i)\right\rangle\left\langle\nu_{1} \otimes \vec{v}(i) \mid \mu_{1}\right\rangle .
\end{aligned}
$$

Very similar arguments give

$$
J_{+} O_{R,(r, s) \vec{\mu}}(Z, Y)=\sum_{T,\left(t^{-}, u^{+}\right) \vec{\nu}}\left(J_{+}\right)_{T,\left(t^{-}, u^{+}\right) \vec{\nu}, R,(r, s) \vec{\mu}} O_{T,\left(t^{-}, u^{+}\right) \vec{\nu}}(Z, Y)
$$

where

$$
\begin{aligned}
\left(J_{+}\right)_{T,\left(t^{-}, u^{+}\right) \vec{\nu}, R,(r, s) \vec{\mu}}= & \sqrt{\frac{\text { hooks }_{r} \text { hooks }_{s}}{\text { hooks }_{t^{-}} \text {hooks }_{u^{+}}}} \\
& \times \sum_{i} \delta_{R T} \delta_{t^{-} r_{i}^{\prime}}(m+1) \frac{d_{s}}{d_{u^{+}}}\left\langle\mu_{2} \otimes \vec{v}(i) \mid \nu_{2}\right\rangle\left\langle\nu_{1} \mid \mu_{1} \otimes \vec{v}(i)\right\rangle \\
= & \sqrt{\frac{\text { hooks }_{\text {hooks }} u^{+}}{\text {hooks }_{t} \text { hooks }_{s}}} \sum_{i} \delta_{R T} \delta_{t^{-} r_{i}^{\prime}}\left\langle\mu_{2} \otimes \vec{v}(i) \mid \nu_{2}\right\rangle\left\langle\nu_{1} \mid \mu_{1} \otimes \vec{v}(i)\right\rangle
\end{aligned}
$$

and

$$
J_{3} O_{R,(r, s) \vec{\mu}}(Z, Y)=\sum_{T,(t, u) \vec{\nu}}\left(J_{3}\right)_{T,(t, u) \vec{\nu}, R,(r, s) \vec{\mu}} O_{T,(t, u) \vec{\nu}}(Z, Y)
$$

where

$$
\left(J_{3}\right)_{T,(t, u) \vec{\nu}, R,(r, s) \vec{\mu}}=\delta_{R T} \delta_{t r} \delta_{u s} \delta_{\vec{\mu} \vec{\nu}}(m-n) .
$$

Our main interest is in the case of 2 rows. This is the simplest setting in which to develop our arguments because in this case there are no multiplicities for the irreducible representations that organize the $Y$ fields. We will make use of a vector $\vec{m}$ which summarizes how to obtain $r$ from $R$. Consider $O_{R,(r, s)}$. The vector $\vec{m}=\left(m_{1}, m_{2}\right)$ tells us how boxes should be removed from $R$ to obtain $r$. Denoting the row lengths of $R$ by $\left(R_{1}, R_{2}\right)$ and of $r$ by $\left(r_{1}, r_{2}\right)$, we have $R_{1}=r_{1}+m_{1}$ and $R_{2}=r_{2}+m_{2}$. As explained in appendix E.1 of [8], we can trade the irreducible representation $s$ organizing the $Y$ fields and $\vec{m}$ for an $\mathrm{SU}(2)$ state. In the new labelling, we specify an operator (which belongs to the sector of the theory constructed using $n Z \mathrm{~s}$ and $m Y \mathrm{~s}$ ) by giving the Young diagram $r$ and an $\mathrm{SU}(2)$ state with labels $\left(j, j_{3}\right)$ where ${ }^{5}$

$$
s=\left(j, j_{3}\right) \quad \longleftrightarrow \quad s_{1}=\frac{m+2 j}{2}, \quad s_{2}=\frac{m-2 j}{2}, \quad j_{3}=\frac{m_{1}-m_{2}}{2} .
$$

We will use the $j, j^{3}$ notation in what follows.

\footnotetext{
${ }^{5} s_{i}$ denote the row lengths of $s$.
} 
We know that $J_{+}$removes a $Z$ box and adds a $Y$ box. Thus, it could have the following possible actions on $r$, the irreducible representation organizing the $Z \mathrm{~s}$ (the box to be removed has a - sign in it - i.e. drop the box with the - sign)

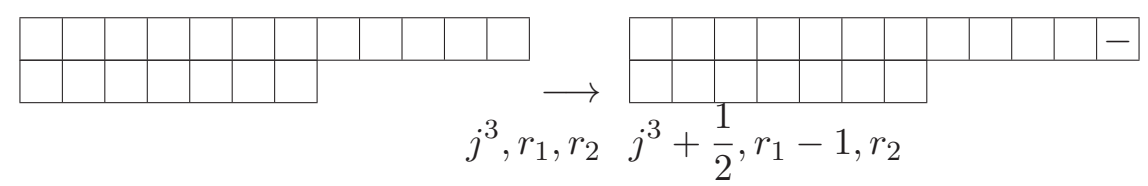

OR

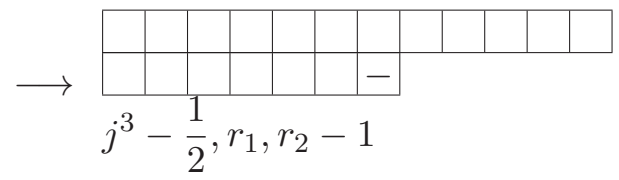

It is trivial to understand how the row lengths $r_{1}$ and $r_{2}$ change when the box shown is dropped. To understand the changes in $j^{3}$, note the following: $J_{+}$does not change the shape of $R$ so that if we know how $r$ changes, we know how $\vec{m}$ changes. In the first possibility above we remove a box from the first row of $r$ which implies that $m_{1}$ grows by 1 and hence that $j^{3}$ grows by $\frac{1}{2}$. In the second possibility above we remove a box from the second row of $r$ which implies that $m_{2}$ grows by 1 and hence that $j^{3}$ decreases by $\frac{1}{2}$. Since we have added a $Y$ box, $J_{+}$can have the following action on $s$ (the box that has been addded has $\mathrm{a}+$ in it)

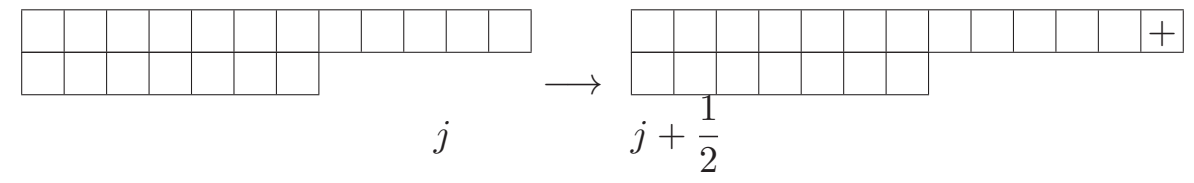

OR

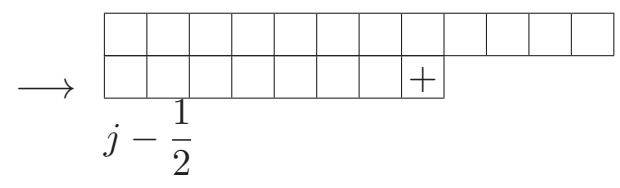

Consequently we have ${ }^{6}$

$$
\begin{aligned}
J_{+} O^{(n, m)}\left(r_{1}, j, j^{3}\right)= & A_{+} O^{(n-1, m+1)}\left(r_{1}-1, j+\frac{1}{2}, j^{3}+\frac{1}{2}\right) \\
& +B_{+} O^{(n-1, m+1)}\left(r_{1}-1, j-\frac{1}{2}, j^{3}+\frac{1}{2}\right) \\
& +C_{+} O^{(n-1, m+1)}\left(r_{1}, j+\frac{1}{2}, j^{3}-\frac{1}{2}\right) \\
& +D_{+} O^{(n-1, m+1)}\left(r_{1}, j-\frac{1}{2}, j^{3}-\frac{1}{2}\right) .
\end{aligned}
$$

\footnotetext{
${ }^{6}$ Note that we don't need to display $r_{2}$ since $r_{2}=n-r_{1}$.
} 
We will describe the computation of $A_{+}$in detail. From (2.23) we have

$$
A_{+}=\sqrt{\frac{\text { hooks }_{r}}{\text { hooks }_{t^{-}}}} \sqrt{\frac{\text { hooks }_{u^{+}}}{\text {hooks }_{s}}}\left(\left\langle j, j^{3} ; \frac{1}{2}, \frac{1}{2} \mid j+\frac{1}{2}, j^{3}+\frac{1}{2}\right\rangle\right)^{2}
$$

where

$$
\begin{aligned}
\sqrt{\frac{\text { hooks }_{r}}{\text { hooks }_{t^{-}}}} & =\sqrt{\frac{\left(r_{1}+1\right)\left(r_{1}-r_{2}\right)}{\left(r_{1}-r_{2}+1\right)}} \\
\sqrt{\frac{\text { hooks }_{u^{+}}}{\text {hooks }_{s}}} & =\sqrt{\frac{m+2 j+42 j+1}{2} \frac{2 j+2}{2 j+2}} \\
\left(\left\langle j, j^{3} ; \frac{1}{2}, \frac{1}{2} \mid j+\frac{1}{2}, j^{3}+\frac{1}{2}\right\rangle\right)^{2} & =\frac{j+j^{3}+1}{2 j+1} .
\end{aligned}
$$

Putting the above factors together, we find

$$
A_{+}=\sqrt{\frac{\left(r_{1}+1\right)\left(r_{1}-r_{2}\right)}{\left(r_{1}-r_{2}+1\right)}} \sqrt{\frac{m+2 j+4}{2} \frac{2 j+1}{2 j+2}} \frac{j+j^{3}+1}{2 j+1} .
$$

In the large $N$ limit this simplifies to

$$
A_{+}=\sqrt{r_{1}} \sqrt{\frac{m+2 j+4}{2} \frac{2 j+1}{2 j+2}} \frac{j+j^{3}+1}{2 j+1} .
$$

Very similar arguments imply that

$$
\begin{aligned}
& B_{+}=\sqrt{r_{1}} \sqrt{\frac{m-2 j+2}{2} \frac{2 j+1}{2 j}} \frac{j-j^{3}}{2 j+1}, \\
& C_{+}=\sqrt{r_{2}} \sqrt{\frac{m+2 j+4}{2} \frac{2 j+1}{2 j+2}} \frac{j-j^{3}+1}{2 j+1}, \\
& D_{+}=\sqrt{r_{2}} \sqrt{\frac{m+2 j+4}{2} \frac{2 j+1}{2 j}} \frac{j+j^{3}}{2 j+1} .
\end{aligned}
$$

Next, consider the action of $J_{-}$. We know that $J_{-}$removes a $Y$ box and adds a $Z$ box. Thus, it could have the following possible actions on $r$, the irreducible representation organizing the $Z \mathrm{~s}$ (the box added has a + sign in it)

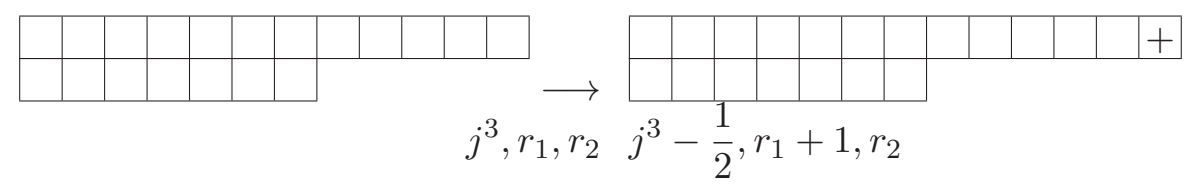

OR

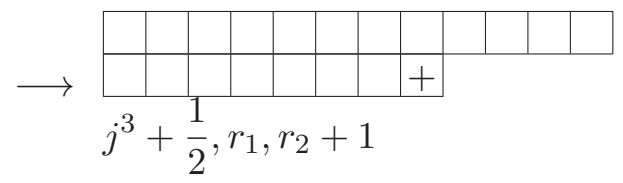


Since we have removed a $Y$ box, $J_{-}$can have the following action on $s$ (the box removed has a - in it)

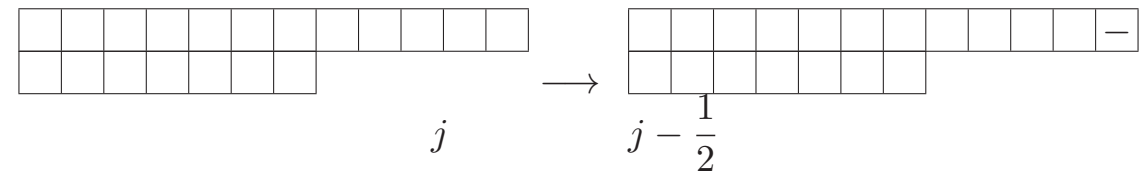

OR

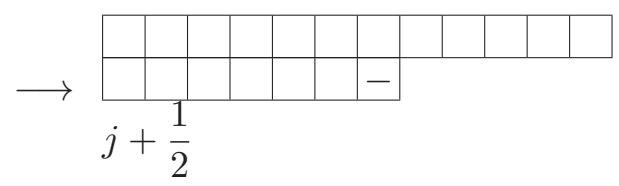

Consequently we have

$$
\begin{aligned}
J_{-} O^{(n, m)}\left(r_{1}, j, j^{3}\right)= & A_{-} O^{(n+1, m-1)}\left(r_{1}+1, j+\frac{1}{2}, j^{3}-\frac{1}{2}\right) \\
& +B_{-} O^{(n+1, m-1)}\left(r_{1}+1, j-\frac{1}{2}, j^{3}-\frac{1}{2}\right) \\
& +C_{-} O^{(n+1, m-1)}\left(r_{1}, j+\frac{1}{2}, j^{3}+\frac{1}{2}\right) \\
& +D_{-} O^{(n+1, m-1)}\left(r_{1}, j-\frac{1}{2}, j^{3}+\frac{1}{2}\right) .
\end{aligned}
$$

To compute $A_{-}$, note that (2.21) implies that

$$
A_{-}=\sqrt{\frac{\text { hooks }_{t^{+}}}{\text {hooks }_{r}}} \sqrt{\frac{\text { hooks }_{s}}{\text { hooks }_{u^{-}}}}\left(\left\langle j, j^{3} \mid \frac{1}{2}, \frac{1}{2} ; j+\frac{1}{2}, j^{3}-\frac{1}{2}\right\rangle\right)^{2}
$$

where

$$
\begin{aligned}
\sqrt{\frac{\text { hooks }_{t^{+}}}{\text {hooks }_{r}}} & =\sqrt{\frac{\left(r_{1}+2\right)\left(r_{1}-r_{2}+1\right)}{\left(r_{1}-r_{2}+2\right)}} \\
\sqrt{\frac{\text { hooks }_{s}}{\text { hooks }_{u^{-}}}} & =\sqrt{\frac{m-2 j}{2} \frac{2 j+2}{2 j+1}} \\
\left(\left\langle j, j^{3} \mid \frac{1}{2}, \frac{1}{2} ; j+\frac{1}{2}, j^{3}-\frac{1}{2}\right\rangle\right)^{2} & =\frac{j-j^{3}+1}{2 j+2} .
\end{aligned}
$$

Thus, we find

$$
A_{-}=\sqrt{\frac{\left(r_{1}+2\right)\left(r_{1}-r_{2}+1\right)}{\left(r_{1}-r_{2}+2\right)}} \sqrt{\frac{m-2 j}{2} \frac{2 j+2}{2 j+1}} \frac{j-j^{3}+1}{2 j+2} .
$$

In the large $N$ limit this becomes

$$
A_{-}=\sqrt{r_{1}} \sqrt{\frac{m-2 j}{2} \frac{2 j+2}{2 j+1}} \frac{j-j^{3}+1}{2 j+2} .
$$


Very similar arguments imply that

$$
\begin{aligned}
& B_{-}=\sqrt{r_{1}} \sqrt{\frac{m+2 j+2}{2} \frac{2 j}{2 j+1}} \frac{j+j^{3}}{2 j}, \\
& C_{-}=\sqrt{r_{2}} \sqrt{\frac{m-2 j}{2} \frac{2 j+2}{2 j+1} \frac{j+j^{3}+1}{2 j+2},} \\
& D_{-}=\sqrt{r_{2}} \sqrt{\frac{m+2 j+2}{2} \frac{2 j}{2 j+1} \frac{j-j^{3}}{2 j} .}
\end{aligned}
$$

Using these results it is straight forward to find

$$
\left[J_{+}, J_{-}\right] O^{(n, m)}\left(r_{1}, j, j^{3}\right)=-n O^{(n, m)}\left(r_{1}, j, j^{3}\right) .
$$

Noting that $J_{3} O^{(n, m)}\left(r_{1}, j, j^{3}\right)=(m-n) O^{(n, m)}\left(r_{1}, j, j^{3}\right)$, this is indeed the correct large $N$ limit of $(2.2)$.

\section{Recursion relations and one loop dilatation operator}

The one loop dilatation operator in the $s u(2)$ sector [5]

$$
D_{2}=-g_{\mathrm{YM}}^{2} \operatorname{Tr}[Y, Z]\left[\partial_{Y}, \partial_{Z}\right]
$$

acting on two giant graviton systems, is given by $[7,8]$

$$
\begin{aligned}
D_{2} O^{(n, m)}\left(r_{1}, j, j^{3}\right)= & g_{Y M}^{2}\left[-\frac{1}{2}\left(m-\frac{(m+2)\left(j^{3}\right)^{2}}{j(j+1)}\right) \Delta O^{(n, m)}\left(r_{1}, j, j^{3}\right)\right. \\
& +\sqrt{\frac{(m+2 j+4)(m-2 j)}{(2 j+1)(2 j+3)} \frac{\left(j+j^{3}+1\right)\left(j-j^{3}+1\right)}{2(j+1)} \Delta O^{(n, m)}\left(r_{1}, j+1, j^{3}\right)} \\
& \left.+\sqrt{\frac{(m+2 j+2)(m-2 j+2)}{(2 j+1)(2 j-1)}} \frac{\left(j+j^{3}\right)\left(j-j^{3}\right)}{2 j} \Delta O^{(n, m)}\left(r_{1}, j-1, j^{3}\right)\right]
\end{aligned}
$$

where $\left(r_{2}=n-r_{1}\right)$

$$
\begin{aligned}
\Delta O^{(n, m)}\left(r_{1}, j, j^{3}\right)= & \sqrt{\left(N+r_{1}\right)\left(N+r_{2}\right)}\left(O^{(n, m)}\left(r_{1}+1, j, j^{3}\right)+O^{(n, m)}\left(r_{1}-1, j, j^{3}\right)\right) \\
& -\left(2 N+r_{1}+r_{2}\right) O^{(n, m)}\left(r_{1}, j, j^{3}\right) .
\end{aligned}
$$

Our goal in this section is to argue that we can recover (3.2) by requiring that the correct algebra

$$
\left[D_{2}, J_{ \pm}\right]=0=\left[D_{2}, J_{3}\right]
$$

is obeyed. We have already obtained a formula for the action of $J_{ \pm}$and $J_{3}$ on restricted Schur polynomials. Our first task is thus to obtain a similar result for the action of $D_{2}$, that can be used in (3.4). We are not trying to write down a detailed formula for $D_{2}$, 
but rather, want to write the general structure of this action that is consistent with the fact that it is derived by summing Feynman diagrams, we are working at large $N$ and the dilatation operator is a hermittian operator. Given this general form, we will derive the detailed matrix elements by requiring (3.4).

There is a pair of derivatives in the one loop dilatation operator (3.1). Since they share an index, their action on the restricted Schur polynomials produces a Kronecker delta function. Equivalently, at one loop our Feynman diagrams have a single interaction vertex and this vertex has two pairs of adjacent fields, $Z, Y$ and $Z^{\dagger}, Y^{\dagger}$. Wick contraction with the vertex will thus set a pair of indices equal, producing a Kronecker delta function. The net consequence of this Kronecker delta function is that the sum over $S_{n+m}$ appearing in the evaluation of $D_{2}$ is reduced to a sum over the subgroup $S_{n+m-1}$ [32]. When we sum over the $S_{n+m-1}$ subgroup, the fundmental orthogonality relation forces one of the representations of $S_{n+m-1}$ subduced by $T$ to be equal to one of the representations subduced by $R$. This allows $D_{2}$ to shift the position of a single box in each of the Young diagram labels of the restricted Schur polynomial. At $p$-loops we will have $p$ insertions of the interaction vertex producing (at most) $p$ Kronecker delta functions, thereby reducing the sum over $S_{n+m}$ to a sum over $S_{n+m-p}$. This allows the $p$-loop dilatation operator to shift the position of (at most) $p$-boxes in each of the Young diagram labels of the restricted Schur polynomial. Returning to one loop, a single box shifts position under the action of $D_{2}$. This implies that we can have the following changes in the labels of our operators

$$
\begin{array}{r}
j^{3} \rightarrow j^{3}, j^{3} \pm 1, \\
j \rightarrow j, j \pm 1, \\
r_{1} \rightarrow r_{1}, r_{1} \pm 1 .
\end{array}
$$

This change of labels implies a total of 27 possible terms under the action of $D_{2}$

$$
D_{2} O^{(n, m)}\left(r_{1}, j, j^{3}\right)=\sum_{c=-1}^{1} \sum_{d=-1}^{1} \sum_{e=-1}^{1} \beta_{r_{1}, j, j^{3}}^{(n, m)}(c, d, e) O^{(n, m)}\left(r_{1}+c, j+d, j^{3}+e\right)
$$

This is slightly too general, as we have not yet put in the constraint that only 1 box can move, i.e. that even if $R$ and $T$ don't agree, by removing a single box from $R$ and a single box from $T$ we can get Young diagrams which do agree. The boxes that must be moved between $R$ and $T$ can be deduced from the boxes moving between $r$ and $t$ and the number of $Y$ boxes that move between the rows (determined by $j^{3}$ ). The matrix element of the dilatation operator that takes

$$
O_{r_{1}, j, j^{3}}^{(n, m)} \longrightarrow O_{r_{1}+a, j+b, j^{3}+c}^{(n, m)} \equiv O_{t_{1}, j^{\prime}, j^{3 \prime}}^{(n, m)}
$$

is $\beta_{r_{1}, j, j^{3}}^{(n, m)}(a, b, c)$. The integer $a$ determines how $r_{1}$ changes, $t_{1}-r_{1}=a$. The integer $c$ determines how $j^{3}$ changes, $j^{3 \prime}-j^{3}=c$. From the definition of $j^{3}$ we have

$$
\begin{aligned}
2 j^{3} & =\left(R_{1}-r_{1}\right)-\left(R_{2}-r_{2}\right), \\
2 j^{3 \prime} & =\left(T_{1}-t_{1}\right)-\left(T_{2}-t_{2}\right) .
\end{aligned}
$$


We also know that $T_{1}+T_{2}=R_{1}+R_{2}=m+n$ and $t_{1}+t_{2}=r_{1}+r_{2}=n$ so that

$$
\begin{aligned}
2 j^{3} & =2 R_{1}-(m+n)-2 r_{1}+n, \\
2 j^{3 \prime} & =2 T_{1}-(m+n)-2 t_{1}+n .
\end{aligned}
$$

Subtracting these last two equations gives

$$
2\left(j^{3 \prime}-j^{3}\right)=2 c=2\left(T_{1}-R_{1}\right)+2\left(r_{1}-t_{1}\right)=2\left(T_{1}-R_{1}\right)-2 a .
$$

Thus, $T_{1}-R_{1}=a+c$ and we must have $|a+c| \leq 1$. This forces

$$
\begin{aligned}
\beta_{r_{1}, j, j^{3}}^{(n, m)}(1,0,1) & =0 & \beta_{r_{1}, j, j^{3}}^{(n, m)}(-1,0,-1) & =0 \\
\beta_{r_{1}, j, j^{3}}^{(n, m)}(1,1,1) & =0 & \beta_{r_{1}, j, j^{3}}^{(n, m)}(-1,1,-1) & =0 \\
\beta_{r_{1}, m, j^{3}}^{(n, m)}(1,-1,1) & =0 & \beta_{r_{1}, j, j^{3}}^{(n, m)}(-1,-1,-1) & =0 .
\end{aligned}
$$

This reduces the number of terms in the action of $D_{2}$ to 21 . Next, we know that the dilatation operator is hermittian $D_{2}=D_{2}^{\dagger}$. This implies that

$$
\left\langle r_{1}+a, j+b, j^{3}+c\left|D_{2}\right| r_{1}, j, j^{3}\right\rangle=\left\langle r_{1}, j, j^{3}\left|D_{2}\right| r_{1}+a, j+b, j^{3}+c\right\rangle .
$$

Further, since

$$
\left\langle r_{1}+a, j+b, j^{3}+c\left|D_{2}\right| r_{1}, j, j^{3}\right\rangle=\beta_{r_{1}, j, j^{3}}^{(n, m)}(a, b, c)
$$

and

$$
\left\langle r_{1}, j, j^{3}\left|D_{2}\right| r_{1}+a, j+b, j^{3}+c\right\rangle=\beta_{r_{1}+a, j+b, j^{3}+c}^{(n, m)}(-a,-b,-c)
$$

we find that the condition $D_{2}=D_{2}^{\dagger}$ implies that

$$
\beta_{r_{1}, j, j^{3}}^{(n, m)}(a, b, c)=\beta_{r_{1}+a, j+b, j^{3}+c}^{(n, m)}(-a,-b,-c) .
$$

This reduces the number of unknown terms to be determined to 11 .

In the large $N$ limit, the string coupling $g_{s}=\frac{1}{N}$ goes to zero. Consequently there is no string splitting or joining. Since each trace in the super Yang-Mills theory corresponds to a closed string state, this translates into the fact that, in the planar limit in the super Yang-Mills theory, different multi-trace structures do not mix. For the open string sector, when the string coupling goes to zero there is again no splitting and joining so that the open string Chan-Paton factors are frozen. The translation of a giant graviton system into an operator in the field theory is straight forward $[8,10-14,33-35]$ : in the operator $O_{r, j, j^{3}}^{(n, m)}$ each row of $r$ corresponds to a giant graviton and each impurity $Y$ corresponds to an open string (this last interpretation is proved in $[8,10]$ ). $j^{3}$ tells us the number of open string end points attached to each giant. Since the Chan-Paton factors are frozen, $j^{3}$ is not changed by the action of the dilatation operator and

$$
\beta(a, b, \pm 1)=0 .
$$

This now leaves 4 unknown terms to be determined. 
Another consequence of working at large $N$ in the displaced corners approximation, is

$$
\beta_{r_{1}+\alpha, j, j^{3}}^{(n, m)}(a, b, c)=\beta_{r_{1}, j, j^{3}}^{(n, m)}(a, b, c)
$$

with $\alpha$ any number of order 1 . This follows because $r_{1}$ is order $N$ and the matrix elements of the dilatation operator depend smoothly on the parameters $r_{1}, j, j^{3}$, so we can replace $r_{1}+\alpha$ by $r_{1}$ making negligible error in the large $N$ limit. There is one point that deserves attention. In general our results depend on $r_{1}, r_{2}$ and on $r_{1}-r_{2}$. Even if $r_{1}=O(N)$ and $r_{2}=O(N)$, if $r_{1}-r_{2}=O(1)$, replacing $r_{1}+\alpha \rightarrow r_{1}$ can result in errors that do not vanish as $N \rightarrow \infty$. In the displaced corners approximation all $r$ row lengths are well separated and this does not happen. It then follows that the $r_{i}$ are conserved and that the coefficients of $\sqrt{r_{1}}$ and $\sqrt{r_{2}}$ in (3.4) must separately vanish. This has a very natural physical interpretation: the $r_{i}$ set the momenta of the giant gravitons and the back reaction on each giant graviton is negligible.

Although we are mainly interested in the dependence of the dilatation operator on $j, j^{3}$, we do know that

$$
\begin{aligned}
\beta_{r_{1}, j, j^{3}}^{(n, m)}( \pm 1, d, e) & =\sqrt{\left(N+r_{1}\right)\left(N+r_{2}\right)} f\left(j, j^{3}, d, e\right) \\
& =\sqrt{\left(N+r_{1}\right)\left(N+n-r_{1}\right)} f\left(j, j^{3}, d, e\right) \\
\beta_{r_{1}, j, j^{3}}^{(n, m)}(0, d, e) & =\left(2 N+r_{1}+r_{2}\right) g\left(j, j^{3}, d, e\right) \\
& =(2 N+n) g\left(j, j^{3}, d, e\right) .
\end{aligned}
$$

These formulas deserve some discussion. The dependence of matrix elements on factors ${ }^{7}$ of boxes in the Young diagram labels has two sources:

1. There is an overall normalization $\sqrt{\frac{f_{T}}{f_{R}}}$. The factors of any boxes that are common to $R$ and $T$ will cancel so that we are left with

$$
F_{1}=\sqrt{\frac{\prod_{i \in \text { boxes in } T \text { that are not in } R} \quad c_{i}}{\prod_{j \in \text { boxes in } R \text { that are not in } T} c_{j}}}
$$

2. When evaluating the dilatation operator, we need to sum over $S_{n+m}$. As discussed above, derivatives with respect to $Y$ and $Z$ produce Kronecker delta functions that restrict the sum to the subgroup $S_{n+m-1}$. The original trace over $R \vdash m+n$ then becomes a trace over an irreducible representation of the subgroup $R^{\prime} \vdash m+n-1$. The sum then produces the factor of the box that must be removed from $R$ to obtain $R^{\prime}$. The trace splits into a trace over $r^{\prime} \vdash n-1$ which sets $r^{\prime}=t^{\prime}$ and a trace over $s$ which depends only on $j, j^{3}$. This dependence is summarized in the functions $f\left(j, j^{3}, d, e\right)$ and $g\left(j, j^{3}, d, e\right)$ above and it is these functions that we want to constrain using the $\mathrm{su}(2)$ invariance.

For the first term in (3.20) we have

$$
F_{1}=\sqrt{\frac{N+r_{1}}{N+r_{2}}} \quad F_{2}=N+r_{2} \quad \text { or } \quad F_{1}=\sqrt{\frac{N+r_{2}}{N+r_{1}}} \quad F_{2}=N+r_{1}
$$

\footnotetext{
${ }^{7}$ Recall that a box in row $i$ and column $j$ has a factor $N-i+j$.
} 
so that

$$
F_{1} \cdot F_{2}=\sqrt{\left(N+r_{1}\right)\left(N+r_{2}\right)}
$$

For the second term in (3.20) we have two contributions which both have $F_{1}=1$ and

$$
F_{2}=N+r_{1} \quad \text { or } \quad F_{2}=N+r_{2}
$$

Thus, the total coefficient of this term is

$$
N+r_{1}+N+r_{2}=2 N+r_{1}+r_{2}=2 N+n
$$

Since we are computing a commutator, the answer for $D_{2}$ will not be unique. Indeed, replacing $D_{2} \rightarrow D_{2}+\alpha \mathbf{1}$ with $\alpha$ a constant, will not change the value of the commutator. To fix the value of $\alpha$ note that there are BPS operators belonging to the $s u(2)$ sector. These operators are annihilated by $D_{2}$, so that the smallest eigenvalue of $D_{2}$ is zero. This fixes $\alpha$.

Now, use

$$
J_{+} O_{r_{1}, j, j^{3}}^{(n, m)}=\sum_{a=-1}^{0} \sum_{b=-\frac{1}{2}}^{\frac{1}{2}} \alpha_{r_{1}, j, j^{3}}^{(n, m)}(a, b) O_{r_{1}+a, j+b, j^{3}-\frac{1}{2}-a}^{(n-1, m+1)}
$$

where (using the results of the last section)

$$
\begin{aligned}
\alpha_{r_{1}, j, j^{3}}^{(n, m)}\left(-1, \frac{1}{2}\right) & =\sqrt{r_{1}} \sqrt{\frac{m+2 j+4}{2}} \frac{j+j^{3}+1}{\sqrt{(2 j+2)(2 j+1)}} \\
\alpha_{r_{1}, j, j^{3}}^{(n, m)}\left(-1,-\frac{1}{2}\right) & =\sqrt{r_{1}} \sqrt{\frac{m-2 j+2}{2}} \frac{j-j^{3}}{\sqrt{2 j(2 j+1)}} \\
\alpha_{r_{1}, j, j^{3}}^{(n, m)}\left(0, \frac{1}{2}\right) & =\sqrt{r_{2}} \sqrt{\frac{m+2 j+4}{2}} \frac{j-j^{3}+1}{\sqrt{(2 j+2)(2 j+1)}} \\
\alpha_{r_{1}, j, j^{3}}^{(n, m)}\left(0,-\frac{1}{2}\right) & =\sqrt{r_{2}} \sqrt{\frac{m-2 j+2}{2}} \frac{j+j^{3}}{\sqrt{2 j(2 j+1)}}
\end{aligned}
$$

and use

$$
D_{2} O_{r_{1}, j, j^{3}}^{(n, m)}=\sum_{a=1}^{-1} \sum_{b=1}^{-1} \sum_{c=1}^{-1} \beta_{r_{1}, j, j^{3}}^{(n, m)}(a, b, c) O_{r_{1}+a, j+b, j^{3}+c}^{(n, m)}
$$

to evaluate

$$
\left[J_{+}, D_{2}\right] O_{r_{1}, j, j^{3}}^{(n, m)}=0 .
$$

The result is

$$
\begin{gathered}
\sum_{a=-1}^{0} \sum_{b=-\frac{1}{2}}^{\frac{1}{2}} \sum_{c=-1}^{1} \sum_{d=-1}^{1} \sum_{e=-1}^{1}\left(\beta_{r_{1}, j, j^{3}}^{(n, m)}(c, d, e) \alpha_{r_{1}+c, j+d, j^{3}+e}^{(n, m)}(a, b)\right. \\
\left.-\alpha_{r_{1}, j, j^{3}}^{(n, m)}(a, b) \beta_{r_{1}+a, j+b, j^{3}-\frac{1}{2}-a}^{(n-1, m+1)}(c, d, e)\right) O_{r_{1}+a+c, j+d+b, j^{3}+e-\frac{1}{2}-a}^{(n-1, m+1)}=0 .
\end{gathered}
$$


The operators $O_{r_{1}, j, j^{3}}^{(n, m)}$ are all linearly independent, so that the coefficient of each term must vanish separately. Further, since $\alpha_{r_{1}, j, j^{3}}^{(n, m)}(-1, \cdot) \propto \sqrt{r_{1}}$ and $\alpha_{r_{1}, j, j^{3}}^{(n, m)}(0, \cdot) \propto \sqrt{r_{2}}$, terms with different values of $a$ in $\alpha_{r_{1}, j, j^{3}}^{(n, m)}(a, \cdot)$ must separately vanish.

To illustrate some of the details, we will discuss some examples of equations that we obtain from (3.33). In particular, we will explain how the $\beta_{r_{1}, j, j^{3}}(0,1,0)$ matrix element is determined. Set $a=0, c=0, e=0, d+b=-\frac{3}{2} \Rightarrow(d, b)=\left(-1,-\frac{1}{2}\right)$ to obtain

$$
\begin{aligned}
\beta_{r_{1}, j, j^{3}}^{(n, m)}(0,-1,0) \alpha_{r_{1}, j-1, j^{3}}^{(n, m)} & \left(0,-\frac{1}{2}\right)-\alpha_{r_{1}, j, j^{3}}^{(n, m)}\left(0,-\frac{1}{2}\right) \beta_{r_{1}, j-\frac{1}{2}, j^{3}-\frac{1}{2}}^{(n-1, m+1)}(0,-1,0)=0, \\
& \sqrt{\frac{m-2 j+4}{2}} \frac{j+j^{3}-1}{\sqrt{(2 j-2)(2 j-1)}} \beta_{r_{1}, j, j^{3}}^{(n, m)}(0,-1,0) \\
& -\sqrt{\frac{m-2 j+2}{2}} \frac{j+j^{3}}{\sqrt{2 j(2 j+1)}} \beta_{r_{1}, j-\frac{1}{2}, j^{3}-\frac{1}{2}}^{(n-1, m+1)}(0,-1,0)=0 .
\end{aligned}
$$

Next, set $a=-1, c=0, e=0, d+b=-\frac{3}{2} \Rightarrow(d, b)=\left(-1,-\frac{1}{2}\right)$ to obtain

$$
\begin{array}{r}
\beta_{r_{1}, j, j^{3}}^{(n, m)}(0,-1,0) \alpha_{r_{1}, j-1, j^{3}}^{(n, m)}\left(-1,-\frac{1}{2}\right)-\alpha_{r_{1}, j, j^{3}}^{(n, m)}\left(-1,-\frac{1}{2}\right) \beta_{r_{1}-1, j-\frac{1}{2}, j^{3}+\frac{1}{2}}^{(n-1, m+1)}(0,-1,0)=0, \\
\sqrt{\frac{m-2 j+4}{2}} \frac{j-j^{3}-1}{\sqrt{(2 j-2)(2 j-1)}} \beta_{r_{1}, j, j^{3}}^{(n, m)}(0,-1,0) \\
-\sqrt{\frac{m-2 j+2}{2}} \frac{j-j^{3}}{\sqrt{(2 j+1) 2 j}} \beta_{r_{1}-1, j-\frac{1}{2}, j^{3}+\frac{1}{2}}^{(n-1, m+1)}(0,-1,0)=0 .
\end{array}
$$

Combining (3.34) and (3.35) we find

$$
\beta_{r_{1}, j, j^{3}}^{(n, m)}(0,-1,0)=\frac{j+j^{3}}{j+j^{3}-1} \frac{j-j^{3}}{j-j^{3}+1} \beta_{r_{1}, j, j^{3}-1}^{(n, m)}(0,-1,0)
$$

which implies that

$$
\beta_{r_{1}, j, j^{3}}^{(n, m)}(0,-1,0) \propto\left(j+j^{3}\right)\left(j-j^{3}\right) .
$$

Daggering equation (3.36) we find

$$
\beta_{r_{1}, j, j^{3}}^{(n, m)}(0,1,0)=\frac{j+j^{3}+1}{j+j^{3}} \frac{j-j^{3}+1}{j-j^{3}+2} \beta_{r_{1}, j, j^{3}-1}^{(n, m)}(0,1,0)
$$

which implies that

$$
\beta_{r_{1}, j, j^{3}}^{(n, m)}(0,1,0) \propto\left(j+j^{3}+1\right)\left(j-j^{3}+1\right) .
$$

Now, set $a=0, b=\frac{1}{2}, c=0, d=1$ and $e=0$ to obtain

$$
\begin{aligned}
& \beta_{r_{1}, j, j^{3}}^{(n, m)}(0,1,0) \alpha_{r_{1}, j+1, j^{3}}^{(n, m)}\left(0, \frac{1}{2}\right)-\alpha_{r_{1}, j, j^{3}}^{(n, m)}\left(0, \frac{1}{2}\right) \beta_{r_{1}, j+\frac{1}{2}, j^{3}-\frac{1}{2}}^{(n-1, m+1)}(0,1,0)=0, \\
& \sqrt{\frac{m+2 j+6}{2}} \frac{j-j^{3}+2}{\sqrt{(2 j+4)(2 j+3)}} \beta_{r_{1}, j, j^{3}}^{(n, m)}(0,1,0) \\
& -\sqrt{\frac{m+2 j+4}{2}} \frac{j-j^{3}+1}{\sqrt{(2 j+1)(2 j+2)}} \beta_{r_{1}, j+\frac{1}{2}, j^{3}-\frac{1}{2}}^{(n-1, m+1)}(0,1,0)=0 .
\end{aligned}
$$


Daggering this we find

$$
\beta_{r_{1}, j-\frac{1}{2}, j^{3}-\frac{1}{2}}^{(n-1, m+1)}(0,-1,0)=\sqrt{\frac{m+2 j+2}{m+2 j}} \frac{j-j^{3}}{j-j^{3}-1} \sqrt{\frac{(2 j-3)(2 j-2)}{(2 j-1) 2 j}} \beta_{r_{1}, j-1, j^{3}}^{(n, m)}(0,-1,0) .
$$

Combining this with (3.34) we find

$$
\begin{aligned}
\beta_{r_{1}, j, j^{3}}^{(n, m)}(0,-1,0)= & \sqrt{\frac{(m-2 j+2)(m+2 j+2)}{(m-2 j+4)(m+2 j)}} \frac{2 j-2}{2 j} \sqrt{\frac{(2 j-1)(2 j-3)}{(2 j+1)(2 j-1)}} \\
& \times \frac{j+j^{3}}{j+j^{3}-1} \frac{j-j^{3}}{j-j^{3}-1} \beta_{r_{1}, j-1, j^{3}}^{(n, m)}(0,-1,0)
\end{aligned}
$$

which implies that

$$
\beta_{r_{1}, j, j^{3}}^{(n, m)}(0,-1,0) \propto \sqrt{\frac{(m+2 j+2)(m-2 j+2)}{(2 j+1)(2 j-1)}} \frac{\left(j+j^{3}\right)\left(j-j^{3}\right)}{2 j}
$$

which is indeed the correct result. Daggering, we find

$$
\beta_{r_{1}, j, j^{3}}^{(n, m)}(0,1,0) \propto \sqrt{\frac{(m+2 j+4)(m-2 j)}{(2 j+1)(2 j+3)}} \frac{\left(j+j^{3}+1\right)\left(j-j^{3}+1\right)}{2(j+1)}
$$

which is also correct. Solving the complete set of recursion relations we find

$$
\begin{aligned}
& D_{2} O^{(n, m)}\left(r_{1}, j, j^{3}\right)= \\
& \sqrt{\frac{(m-2 j+2)(m+2 j+2)}{(2 j+1)(2 j-1)}} \frac{\left(j+j^{3}\right)\left(j-j^{3}\right)}{2 j}\left[c_{010}\left(2 N+r_{1}+r_{2}\right) O^{(n, m)}\left(r_{1}, j-1, j^{3}\right)\right. \\
& \left.+c_{110} \sqrt{\left(N+r_{1}\right)\left(N+r_{2}\right)}\left(O^{(n, m)}\left(r_{1}-1, j-1, j^{3}\right)+O^{(n, m)}\left(r_{1}+1, j-1, j^{3}\right)\right)\right] \\
& +\sqrt{\frac{(m+2 j+4)(m-2 j)}{(2 j+3)(2 j+1)}} \frac{\left(j+j^{3}+1\right)\left(j-j^{3}+1\right)}{2 j+2}\left[c_{010}\left(2 N+r_{1}+r_{2}\right) O^{(n, m)}\left(r_{1}, j+1, j^{3}\right)\right. \\
& \left.+c_{110} \sqrt{\left(N+r_{1}\right)\left(N+r_{2}\right)}\left(O^{(n, m)}\left(r_{1}-1, j+1, j^{3}\right)+O^{(n, m)}\left(r_{1}+1, j+1, j^{3}\right)\right)\right] \\
& +\left(-\frac{1}{2}\left(m-\frac{(m+2)\left(j^{3}\right)^{2}}{j(j+1)}\right)\right)\left[c_{010}\left(2 N+r_{1}+r_{2}\right) O^{(n, m)}\left(r_{1}, j, j^{3}\right)\right. \\
& \left.+c_{110} \sqrt{\left(N+r_{1}\right)\left(N+r_{2}\right)}\left(O^{(n, m)}\left(r_{1}-1, j, j^{3}\right)+O^{(n, m)}\left(r_{1}+1, j, j^{3}\right)\right)\right]
\end{aligned}
$$

where $c_{010}$ and $c_{110}$ are arbitrary constants, independent of $j, j^{3}$ and $r_{1}$. Thus, we have determined the $j, j^{3}$ dependence of the matrix elements of the one loop dilatation operator. Achieving this at higher loops is one of the main goals of this article. To completely determine the spectrum of anomalous dimensions, we need to determine the constants $c_{010}$ and $c_{110}$ in the above expression. These constants are tightly constrained as we now explain. In the large $N$ regime, we can take a continuum limit of the action of the dilatation operator. Towards this end, introduce the continuous variable $\rho=\frac{r_{1}-r_{2}}{2 \sqrt{N+r_{2}}}$ and replace $O^{(r, m)}\left(r_{1}, j, j^{3}\right)$ with $O^{(r, m)}\left(\rho, j, j^{3}\right) . r_{1}$ is the longer (top) row and $r_{2}$ is the shorter bottom 
row. When $\rho$ is order 1 the dilatation operator becomes an $N$ independent differential operator [9]. Expanding we have

$$
\sqrt{\left(N+r_{1}\right)\left(N+r_{2}\right)}=\left(N+r_{2}\right)\left(1+\frac{1}{2} \frac{r_{1}-r_{2}}{N+r_{2}}-\frac{1}{8} \frac{\left(r_{1}-r_{2}\right)^{2}}{\left(N+r_{2}\right)^{2}}+\ldots\right)
$$

The first term above is $O(N)$, the second $O(\sqrt{N})$ and the third $O(1)$.

$$
\begin{aligned}
O^{(n, m)}\left(\rho-\frac{1}{\sqrt{N+r_{2}}}, j, j^{3}\right)= & O^{(n, m)}\left(\rho, j, j^{3}\right)-\left.\frac{1}{\sqrt{N+r_{2}}} \frac{\partial O^{(n, m)}}{\partial \rho}\right|_{\rho, j, j^{3}} \\
& +\left.\frac{1}{N+r_{2}} \frac{\partial^{2} O^{(n, m)}}{\partial \rho^{2}}\right|_{\rho, j, j^{3}}+\ldots
\end{aligned}
$$

These expansions are only valid if $r_{1}-r_{2} \ll N+r_{2}$, which is certainly not always the case. However, we will learn something about the relation between the coeficients $c_{110}$ and $c_{010}$ by studying this situation. Using these expansions we have

$$
\begin{aligned}
& c_{010}\left(2 N+r_{1}+r_{2}\right) O^{(n, m)}\left(r_{1}, j, j^{3}\right) \\
& +c_{110} \sqrt{\left(N+r_{1}\right)\left(N+r_{2}\right)}\left(O^{(n, m)}\left(r_{1}-1, j, j^{3}\right)+O^{(n, m)}\left(r_{1}+1, j, j^{3}\right)\right) \\
= & {\left[c_{110}+2 c_{010}\right]\left(N+r_{2}\right) O^{(n, m)}\left(r_{1}, j, j^{3}\right)+\frac{1}{2}\left[c_{110}+2 c_{010}\right] \sqrt{N+r_{2}} O^{(n, m)}\left(r_{1}, j, j^{3}\right)+O(1) }
\end{aligned}
$$

Again, the lowest eigenvalue of this operator is zero, reflecting a BPS operator. To achieve this, the $O(N)$ and $O(\sqrt{N})$ pieces of this expansion must cancel which determines $c_{110}+$ $2 c_{010}=0$. Thus, up to an overall normalization which our argument can't determine, we have reproduced (3.2).

\section{Continuum limit}

We have demonstrated that the requirement that the one loop dilatation operator closes the correct Lie algebra when commuted with an $s u(2)$ subgroup of the $\mathcal{R}$-symmetry group determines a set of recursion relations. Solving these recursion relations we have recovered the formula for the one loop dilatation operator derived in $[7,8]$ by detailed computation. We are interested in carrying this analysis out at higher loops. The resulting recursion relations become very clumsy to solve. To overcome this difficulty, we will now pursue a continuum approach to the problem, replacing the discrete variables $j, j^{3}$ by continuous variables $x_{j}, x_{j^{3}}$. The advantage of considering a continuum limit is that our recursion relations will be replaced by partial differential equations and we are able to explicitely determine the general solution of these partial differential equations. In this section we will motivate the continuum limit we study by considering the dilatation operator eigenproblem at one loop.

The structure of the action of the one loop dilatation operator problem given in (3.2) exhibits an interesting factorization. There is an action of $\Delta$ which acts only on the $r$ label times an action that is only on the $j, j^{3}$ labels. The continuum limit we consider here is 
concerned with the action on the $j, j^{3}$ labels. Recall that we take $m$ to be $O(\sqrt{N})$. The discrete eigenproblem that we consider is $[7,8]$

$$
\begin{aligned}
-\lambda \psi\left(j, j^{3}\right)= & \sqrt{\frac{(m+2 j+4)(m-2 j)}{(2 j+1)(2 j+3)}} \frac{\left(j+j^{3}+1\right)\left(j-j^{3}+1\right)}{2(j+1)} \psi\left(j+1, j^{3}\right) \\
& \sqrt{\frac{(m+2 j+2)(m-2 j+2)}{(2 j+1)(2 j-1)}} \frac{\left(j+j^{3}\right)\left(j-j^{3}\right)}{2 j} \psi\left(j-1, j^{3}\right) \\
& -\frac{1}{2}\left(m-\frac{(m+2)\left(j^{3}\right)^{2}}{j(j+1)}\right) \psi\left(j, j^{3}\right) .
\end{aligned}
$$

The variables that become continuous as we take $N \rightarrow \infty$ are

$$
x_{j}=\frac{j}{\sqrt{m}}, \quad x_{j^{3}}=\frac{j^{3}}{\sqrt{m}}
$$

Replace $\psi\left(j, j^{3}\right)$ by $\psi\left(x_{j}, x_{j^{3}}\right)$ and use the expansions

$$
\begin{aligned}
& -\frac{1}{2}\left(m-\frac{(m+2)\left(j^{3}\right)^{2}}{j(j+1)}\right)=-\frac{m}{2}+\frac{m}{2} \frac{x_{j^{3}}^{2}}{x_{j}^{2}}-\frac{\sqrt{m}}{2} \frac{x_{j^{3}}^{2}}{x_{j}^{3}}+\frac{x_{j^{3}}^{2}}{2 x_{j}^{4}}+\frac{x_{j^{3}}^{2}}{x_{j}^{2}} \\
& \sqrt{\frac{(m+2 j+4)(m-2 j)}{(2 j+1)(2 j+3)} \frac{\left(j+j^{3}+1\right)\left(j-j^{3}+1\right)}{2(j+1)}}= \\
& \sqrt{\frac{m}{4}+\frac{1}{2}-\frac{x_{j}^{2}}{2}+\frac{1}{32 x_{j}^{2}}-\frac{m}{4} \frac{x_{j^{3}}^{2}}{x_{j}^{2}}-\frac{1}{2} \frac{x_{j^{3}}^{2}}{x_{j}^{2}}+\frac{x_{j^{3}}^{2}}{2}-\frac{25 x_{j^{3}}^{2}}{32 x_{j}^{4}}+\frac{\sqrt{m} x_{j^{3}}^{2}}{2 x_{j}^{3}}} \\
& \frac{m}{4}+\frac{1}{2}-\frac{x_{j}^{2}}{2}+\frac{1}{32 x_{j}^{2}}-\frac{m}{4} \frac{x_{j^{3}}^{2}}{x_{j}^{2}}-\frac{1}{2} \frac{x_{j^{3}}^{2}}{x_{j}^{2}}+\frac{x_{j^{3}}^{2}}{2}-\frac{x_{j^{3}}^{2}}{32 x_{j}^{4}} .
\end{aligned}
$$

It is now a simple matter to find the following eigenproblem in the continuum

$$
\frac{1}{4}\left(1-\frac{x_{j^{3}}^{2}}{x_{j}^{2}}\right) \frac{d^{2} \psi}{d x_{j}^{2}}+\frac{x_{j^{3}}^{2}}{2 x_{j}^{3}} \frac{d \psi}{d x_{j}}+\left[-\frac{5 x_{j^{3}}^{2}}{16 x_{j}^{4}}+1-x_{j}^{2}+\frac{1}{16 x_{j}^{2}}+x_{j^{3}}^{2}\right] \psi=-\lambda \psi .
$$

In obtaining this result the form for our continuum limit, as spelled out in (4.2) is crucial. Indeed, if one sets $x_{j}=j / m^{\alpha}$ the "kinetic" and "harmonic potential" terms on the l.h.s. are only the same size if $\alpha=\frac{1}{2}$. Now, set $\psi=\sqrt{x_{j}} g$ to obtain

$$
\frac{1}{4}\left(1-\frac{x_{j^{3}}^{2}}{x_{j}^{2}}\right) \frac{d^{2} g}{d x_{j}^{2}}+\frac{1}{4 x_{j}}\left(1+\frac{x_{j^{3}}^{2}}{2 x_{j}^{3}}\right) \frac{d g}{d x_{j}}+\left[1-x_{j}^{2}+x_{j^{3}}^{2}\right] g=-\lambda g .
$$

Finally, in terms of the new variable $u$ defined by $u^{2}=x_{j}^{2}-x_{j^{3}}^{2}$ we find

$$
\frac{1}{4} \frac{d^{2} g}{d u^{2}}+\frac{1}{4 u} \frac{d g}{d u}+\left(1-u^{2}\right) g=-\lambda g .
$$


If we set $r=2 u$ we find the eigenproblem of the 2-dimensional oscillator with zero angular momentum. The energy spacing is 2 (recall $j \geq 0$ to see this). This is exactly the spectrum obtained by solving the discrete problem $[7,8]$. It is also easy to check that the eigenvectors of the discrete problem are in perfect agreement with the eigenfunctions of (4.8). Thus, the continuum problem contains the same information as the discrete problem.

To get the correct spectrum we must obtain the $O(m), O(\sqrt{m})$ and $O(1)$ pieces of the matrix elements of the dilatation operator. Writing things schematically, we should expand our dilatation operator matrix elements as

$$
\beta=m f^{(0)}+\sqrt{m} f^{(1)}+f^{(2)}+\frac{f^{(3)}}{\sqrt{m}}+O\left(\frac{1}{m}\right)
$$

and we should expand

$$
\alpha=\sqrt{m} \alpha^{(0)}+\alpha^{(1)}+\frac{1}{\sqrt{m}} \alpha^{(2)}+\frac{1}{m} \alpha^{(3)}+O\left(\frac{1}{m^{\frac{3}{2}}}\right)
$$

After expansion (3.4) gives 3 sets of non-trivial equations, and these three equations are the complete content of the recursion relations. They are obtained by plugging the above expansions into (3.4) and setting the coefficients of $m, \sqrt{m}$ and 1 to zero. The terms with coefficient $m^{\frac{3}{2}}$ trivially vanish. The terms with negative powers of $m$ also do not give new equations: they vanish automatically because we are working in the $m=\sqrt{N} \rightarrow \infty$ limit.

At one loop, solving the partial differential equations that arise from (3.4) must reproduce the following expansions

$$
\begin{aligned}
\beta_{r_{1}, j, j^{3}}^{(n, m)}(c, 0,0) & =-\frac{m}{2}+\frac{m}{2} \frac{x_{j^{3}}^{2}}{x_{j}^{2}}-\frac{\sqrt{m}}{2} \frac{x_{j^{3}}^{2}}{x_{j}^{3}}+\frac{x_{j^{3}}^{2}}{2 x_{j}^{4}}+\frac{x_{j^{3}}^{2}}{x_{j}^{2}} \\
\beta_{r_{1}, j, j^{3}}^{(n, m)}(c, 1,0) & =\frac{m}{4}+\frac{1}{2}-\frac{x_{j}^{2}}{2}+\frac{1}{32 x_{j}^{2}}-\frac{m}{4} \frac{x_{j^{3}}^{2}}{x_{j}^{2}}-\frac{1}{2} \frac{x_{j^{3}}^{2}}{x_{j}^{2}}+\frac{x_{j^{3}}^{2}}{2}-\frac{25 x_{j^{3}}^{2}}{32 x_{j}^{4}}+\frac{\sqrt{m} x_{j^{3}}^{2}}{2 x_{j}^{3}} \\
\beta_{r_{1}, j, j^{3}}^{(n, m)}(c,-1,0) & =\frac{m}{4}+\frac{1}{2}-\frac{x_{j}^{2}}{2}+\frac{1}{32 x_{j}^{2}}-\frac{m}{4} \frac{x_{j^{3}}^{2}}{x_{j}^{2}}-\frac{1}{2} \frac{x_{j^{3}}^{2}}{x_{j}^{2}}+\frac{x_{j^{3}}^{2}}{2}-\frac{x_{j^{3}}^{2}}{32 x_{j}^{4}}
\end{aligned}
$$

Given these continuum results, we can immediately claim that we have reproduced (3.2). Indeed, the ambiguity in reconstructing the exact functions $\beta_{r_{1}, j, j^{3}}^{(n, m)}(c, d, 0)$ of the discrete variables $j, j^{3}$ from the continuum expressions above is order $\frac{1}{m}$ and we are working in the $m=\sqrt{N} \rightarrow \infty$ limit.

Finally, it is important to note that the solutions to our continuum differential equations are not unique. Indeed, we are finding a dilatation operator $D$ that obeys

$$
\left[J_{ \pm}, D\right]=0=\left[J_{3}, D\right] .
$$

Given a first solution, another solution is easily constructed by rescaling and shifting

$$
D \rightarrow \kappa_{1} D+2 k_{0} \mathbf{1}
$$

where $\mathbf{1}$ is the identity. Thus, there will always be two arbitrary constants in our solutions. This has important implications for us, particularly when it comes to finding the 
most general solution to the partial differential equations we will derive. For example, by choosing $\kappa_{1}=\frac{1}{\sqrt{m}} \gamma$ we see that we shift

$$
\begin{aligned}
& \beta=m f^{(0)}+\sqrt{m} f^{(1)}+f^{(2)}+\frac{f^{(3)}}{\sqrt{m}}+O\left(\frac{1}{m}\right) \longrightarrow \\
& \beta^{\prime}=m f^{(0)}+\sqrt{m}\left(f^{(1)}+\gamma f^{(0)}\right)+f^{(2)}+\gamma f^{(1)}+\frac{f^{(3)}+\gamma f^{(2)}}{\sqrt{m}}+O\left(\frac{1}{m}\right)
\end{aligned}
$$

In what follows, we will construct the solution that has $\gamma=0$ and say that "we have the most general solution up to symmetry". Note that by choosing $\kappa_{1}=\frac{1}{m} \gamma$ we would have

$$
\beta^{\prime}=m f^{(0)}+\sqrt{m} f^{(1)}+f^{(2)}+\gamma f^{(0)}+\frac{f^{(3)}+\gamma f^{(1)}}{\sqrt{m}}+O\left(\frac{1}{m}\right) .
$$

We will thus also not include terms $\propto f^{(0)}$ when solving the partial differential equations that determine $f^{(2)}$. This completes out discussion of the continuum limit.

\section{Differential equations and higher loop anomalous dimensions}

The main goal of this section is to study the constraints implied by (3.4) on the $p$-loop dilatation operator. As we discussed above, the $p$-loop dilatation operator allows a total of $p$ boxes on the Young diagram labels of the restricted Schur polynomial to move. In this case, the requirement that $J_{+}$commutes with $D$ implies that

$$
\begin{array}{r}
\sum_{b=-\frac{1}{2}}^{\frac{1}{2}} \sum_{d=-p}^{p}\left[\beta_{r_{1}, j, j^{3}}^{(n, m)}(c, d, 0) \alpha_{r_{1}+c, j+d, j^{3}}^{(n, m)}(a, b)\right. \\
\left.-\alpha_{r_{1}, j, j^{3}}^{(n, m)}(a, b) \beta_{r_{1}+a, j+b, j^{3}-\frac{1}{2}-a}^{(n-1, m+1)}(c, d, 0)\right] O_{r_{1}+a+c, j+d+b, j^{3}-\frac{1}{2}-a}^{(n-1, m+1)}=0
\end{array}
$$

which can be rewritten as

$$
\begin{gathered}
\beta_{r_{1}, j, j^{3}}^{(n, m)}(c, d, 0) \alpha_{r_{1}+c, j+d, j^{3}}^{(n, m)}\left(a, \frac{1}{2}\right)+\beta_{r_{1}, j, j^{3}}^{(n, m)}(c, d+1,0) \alpha_{r_{1}+c, j+d+1, j^{3}}^{(n, m)}\left(a,-\frac{1}{2}\right) \\
-\alpha_{r_{1}, j, j^{3}}^{(n, m)}\left(a, \frac{1}{2}\right) \beta_{r_{1}+a, j+\frac{1}{2}, j^{3}-\frac{1}{2}-a}^{(n-1, m+1)}(c, d, 0)-\alpha_{r_{1}, j, j^{3}}^{(n, m)}\left(a,-\frac{1}{2}\right) \beta_{r_{1}+a, j-\frac{1}{2}, j^{3}-\frac{1}{2}-a}^{(n-1, m+1)}(c, d+1,0)=0 .
\end{gathered}
$$

Recall that $\alpha(-1, \cdot) \propto \sqrt{r_{1}}$ and $\alpha(0, \cdot) \propto \sqrt{r_{2}}$ so that we get independent equations from (5.1) for each value of $a=\{-1,0\}, c=\{-p,-p+1, \cdots, p-1, p\}$, and $d+b$ where $b= \pm \frac{1}{2}$ and $d=\{-p,-p+1, \cdots, p-1, p\}$. We will freely make use of the result of the appendix in this section.

To begin we will consider $a=0$ in (5.2). A few words on how we perform the expansion of the $\alpha_{r_{1}, j, j^{3}}\left(a, \pm \frac{1}{2}\right)$ is in order. After rewriting $j, j^{3}$ in terms of $x_{j}, x_{j^{3}}$

$$
\begin{aligned}
\alpha_{r_{1}, j j^{3}}\left(0, \frac{1}{2}\right) & =\sqrt{r_{2}} \sqrt{\frac{m+2 j+4}{2}} \frac{j-j^{3}+1}{\sqrt{2 j+2} \sqrt{2 j+1}} \\
& =\sqrt{r_{2}} \sqrt{\frac{m}{2}} \sqrt{1+2 \frac{x_{j}}{\sqrt{m}}+\frac{4}{m}} \frac{x_{j}-x_{j^{3}}+\frac{1}{\sqrt{m}}}{\sqrt{2 x_{j}+\frac{2}{\sqrt{m}}} \sqrt{2 x_{j}+\frac{1}{\sqrt{m}}}}
\end{aligned}
$$


we perform an expansion treating $\frac{1}{\sqrt{m}}$ and $\frac{1}{m}$ as small numbers. Using these expansions, after equating the coefficients of $m^{\frac{3}{2}}$ to zero, in

$$
\begin{gathered}
\beta_{r_{1}, j, j^{3}}^{(n, m)}(c, d, 0) \alpha_{r_{1}+c, j+d, j^{3}}^{(n, m)}\left(0, \frac{1}{2}\right)-\beta_{r_{1}, j+\frac{1}{2}, j^{3}-\frac{1}{2}}^{(n-1, m+1)}(c, d, 0) \alpha_{r_{1}, j, j}^{(n, m)}\left(0, \frac{1}{2}\right) \\
+\beta_{r_{1}, j, j^{3}}^{(n, m)}(c, d+1,0) \alpha_{r_{1}+c, j+d+1, j^{3}}^{(n, m)}\left(0,-\frac{1}{2}\right)-\beta_{r_{1}, j-\frac{1}{2}, j^{3}-\frac{1}{2}}^{(n-1, m+1)}(c, d+1,0) \alpha_{r_{1}, j, j^{3}}^{(n, m)}\left(0,-\frac{1}{2}\right)=0
\end{gathered}
$$

we find

$$
\begin{gathered}
\quad \frac{\left(x_{j}-x_{j^{3}}\right)}{2 \sqrt{2} x_{j}} f_{c, d}^{(0)}\left(x_{j}, x_{j^{3}}\right)+\frac{\left(x_{j}+x_{j^{3}}\right)}{2 \sqrt{2} x_{j}} f_{c, d+1}^{(0)}\left(x_{j}, x_{j^{3}}\right) \\
-\frac{\left(x_{j}-x_{j^{3}}\right)}{2 \sqrt{2} x_{j}} f_{c, d}^{(0)}\left(x_{j}, x_{j^{3}}\right)-\frac{\left(x_{j}+x_{j^{3}}\right)}{2 \sqrt{2} x_{j}} f_{c, d+1}^{(0)}\left(x_{j}, x_{j^{3}}\right)=0
\end{gathered}
$$

which is trivially obeyed. By equating the $O(m)$ term to zero we have

$$
\begin{aligned}
2 x_{j^{3}}\left(d f_{c, d}^{(0)}-(d+1) f_{c, d+1}^{(0)}\right)+ & x_{j}\left(x_{j}\left(\frac{\partial f_{c, d}^{(0)}}{\partial x_{j^{3}}}+\frac{\partial f_{c, d+1}^{(0)}}{\partial x_{j^{3}}}-\frac{\partial f_{c, d}^{(0)}}{\partial x_{j}}+\frac{\partial f_{c, d+1}^{(0)}}{\partial x_{j}}\right)\right. \\
& \left.+x_{j^{3}}\left(-\frac{\partial f_{c, d}^{(0)}}{\partial x_{j^{3}}}+\frac{\partial f_{c, d+1}^{(0)}}{\partial x_{j^{3}}}+\frac{\partial f_{c, d}^{(0)}}{\partial x_{j}}+\frac{\partial f_{c, d+1}^{(0)}}{\partial x_{j}}\right)\right)=0
\end{aligned}
$$

Equating the $O(\sqrt{m})$ term to zero gives

$$
\begin{aligned}
& x_{j}\left(2 d\left(4 x_{j}^{2}-1\right)\left(f_{c, d}^{(0)}-f_{c, d+1}^{(0)}\right)-x_{j}\left[x _ { j } \left(\frac{\partial^{2} f_{c, d}^{(0)}}{\partial x_{j^{3}}^{2}}+\frac{\partial^{2} f_{c, d+1}^{(0)}}{\partial x_{j^{3}}^{2}}-4 \frac{\partial f_{c, d}^{(1)}}{\partial x_{j^{3}}}-4 \frac{\partial f_{c, d+1}^{(1)}}{\partial x_{j^{3}}}\right.\right.\right. \\
& \left.\left.-2 \frac{\partial^{2} f_{c, d}^{(0)}}{\partial x_{j} \partial x_{j^{3}}}+2 \frac{\partial^{2} f_{c, d+1}^{(0)}}{\partial x_{j} \partial x_{j^{3}}}\right)-\frac{\partial f_{c, d}^{(0)}}{\partial x_{j^{3}}}+\frac{\partial f_{c, d+1}^{(0)}}{\partial x_{j^{3}}}+\frac{\partial f_{c, d}^{(0)}}{\partial x_{j}}+\frac{\partial f_{c, d+1}^{(0)}}{\partial x_{j}}\right]+x_{j}^{2}\left(-\left[4 x _ { j } \left(-\frac{\partial f_{c, d}^{(0)}}{\partial x_{j^{3}}}\right.\right.\right. \\
& \left.+\frac{\partial f_{c, d+1}^{(0)}}{\partial x_{j^{3}}}+\frac{\partial f_{c, d}^{(0)}}{\partial x_{j}}+\frac{\partial f_{c, d+1}^{(0)}}{\partial x_{j}}\right)+8 f_{c, d}^{(0)}+16 f_{c, d+1}^{(0)}+4 \frac{\partial f_{c, d}^{(1)}}{\partial x_{j}}-4 \frac{\partial f_{c, d+1}^{(1)}}{\partial x_{j}}+\frac{\partial^{2} f_{c, d}^{(0)}}{\partial x_{j}^{2}} \\
& \left.\left.\left.+\frac{\partial^{2} f_{c, d+1}^{(0)}}{\partial x_{j}^{2}}\right]\right)+2 f_{c, d+1}^{(0)}\right)-x_{j^{3}}\left(x _ { j } \left[-8 d f_{c, d}^{(1)}+8 d f_{c, d+1}^{(1)}-3 \frac{\partial f_{c, d}^{(0)}}{\partial x_{j^{3}}}+\frac{\partial f_{c, d+1}^{(0)}}{\partial x_{j^{3}}}+8 f_{c, d+1}^{(1)}\right.\right. \\
& \left.+3 \frac{\partial f_{c, d}^{(0)}}{\partial x_{j}}+\frac{\partial f_{c, d+1}^{(0)}}{\partial x_{j}}\right]+4(2 d+3)\left[d f_{c, d}^{(0)}-(d+1) f_{c, d+1}^{(0)}\right]+x_{j}^{2}\left[-\frac{\partial^{2} f_{c, d}^{(0)}}{\partial x_{j^{3}}^{2}}+\frac{\partial^{2} f_{c, d+1}^{(0)}}{\partial x_{j^{3}}^{2}}+4 \frac{\partial f_{c, d}^{(1)}}{\partial x_{j^{3}}}\right. \\
& -4 \frac{\partial f_{c, d+1}^{(1)}}{\partial x_{j^{3}}}+2 \frac{\partial^{2} f_{c, d}^{(0)}}{\partial x_{j} \partial x_{j^{3}}}+2 \frac{\partial^{2} f_{c, d+1}^{(0)}}{\partial x_{j} \partial x_{j^{3}}}-8 f_{c, d}^{(0)}+8 f_{c, d+1}^{(0)}-4 \frac{\partial f_{c, d}^{(1)}}{\partial x_{j}}-4 \frac{\partial f_{c, d+1}^{(1)}}{\partial x_{j}} \\
& \left.\left.-\frac{\partial^{2} f_{c, d}^{(0)}}{\partial x_{j}^{2}}+\frac{\partial^{2} f_{c, d+1}^{(0)}}{\partial x_{j}^{2}}\right]+4 x_{j}^{3}\left(\frac{\partial f_{c, d}^{(0)}}{\partial x_{j^{3}}}+\frac{\partial f_{c, d+1}^{(0)}}{\partial x_{j^{3}}}-\frac{\partial f_{c, d}^{(0)}}{\partial x_{j}}+\frac{\partial f_{c, d+1}^{(0)}}{\partial x_{j}}\right)\right)=0
\end{aligned}
$$


Finally, equating the $O(1)$ term to zero we find another equation that is rather long and hence we will not quote it here. We will also study the equations obtained by plugging $a=-1$ into (5.2). Equating the term in

$$
\begin{gathered}
\beta_{r_{1}, j, j^{3}}^{(n, m)}(c, d, 0) \alpha_{r_{1}+c, j+d, j^{3}}^{(n, m)}\left(-1, \frac{1}{2}\right)-\beta_{r_{1}-1, j+\frac{1}{2}, j^{3}+\frac{1}{2}}^{(n-1, m+1)}(c, d, 0) \alpha_{r_{1}, j, j}^{(n, m)}\left(-1, \frac{1}{2}\right) \\
+\beta_{r_{1}, j, j^{3}}^{(n, m)}(c, d+1,0) \alpha_{r_{1}+c, j+d+1, j^{3}}^{(n, m)}\left(-1,-\frac{1}{2}\right)-\beta_{r_{1}-1, j-\frac{1}{2}, j^{3}+\frac{1}{2}}^{(n-1, m+1)}(c, d+1,0) \alpha_{r_{1}, j, j^{3}}^{(n, m)}\left(-1,-\frac{1}{2}\right)=0
\end{gathered}
$$

of order $m^{3 / 2}$ to zero, we find the equation

$$
\begin{gathered}
\quad \frac{\left(x_{j}+x_{j^{3}}\right)}{2 \sqrt{2} x_{j}} f_{c, d}^{(0)}\left(x_{j}, x_{j^{3}}\right)+\frac{\left(x_{j}-x_{j^{3}}\right)}{2 \sqrt{2} x_{j}} f_{c, d+1}^{(0)}\left(x_{j}, x_{j^{3}}\right) \\
-\frac{\left(x_{j}+x_{j^{3}}\right)}{2 \sqrt{2} x_{j}} f_{c, d}^{(0)}\left(x_{j}, x_{j^{3}}\right)-\frac{\left(x_{j}-x_{j^{3}}\right)}{2 \sqrt{2} x_{j}} f_{c, d+1}^{(0)}\left(x_{j}, x_{j^{3}}\right)=0
\end{gathered}
$$

that is again trivially obeyed. The coefficient of the term of order $m$ is

$$
\begin{array}{r}
-x_{j}^{2}\left(\frac{\partial f_{c, d}^{(0)}}{\partial x_{j^{3}}}+\frac{\partial f_{c, d+1}^{(0)}}{\partial x_{j^{3}}}+\frac{\partial f_{c, d}^{(0)}}{\partial x_{j}}-\frac{\partial f_{c, d+1}^{(0)}}{\partial x_{j}}\right) \\
-x_{j^{3}}\left(2 d f_{c, d}^{(0)}-2(d+1) f_{c, d+1}^{(0)}+x_{j}\left(\frac{\partial f_{c, d}^{(0)}}{\partial x_{j^{3}}}-\frac{\partial f_{c, d+1}^{(0)}}{\partial x_{j^{3}}}+\frac{\partial f_{c, d}^{(0)}}{\partial x_{j}}+\frac{\partial f_{c, d+1}^{(0)}}{\partial x_{j}}\right)\right)=0
\end{array}
$$

From the coefficient of the $O(\sqrt{m})$ term we find

$$
\begin{array}{r}
x_{j}\left(-x_{j}^{2}\left[-8(d-1) f_{c, d}^{(0)}+8(d+2) f_{c, d+1}^{(0)}+\frac{\partial^{2} f_{c, d}^{(0)}}{\partial x_{j^{3}}^{2}}+\frac{\partial^{2} f_{c, d+1}^{(0)}}{\partial x_{j^{3}}^{2}}+4 \frac{\partial f_{c, d}^{(1)}}{\partial x_{j^{3}}}+4 \frac{\partial f_{c, d+1}^{(1)}}{\partial x_{j^{3}}}\right.\right. \\
\left.+2 \frac{\partial^{2} f_{c, d}^{(0)}}{\partial x_{j} \partial x_{j^{3}}}-2 \frac{\partial^{2} f_{c, d+1}^{(0)}}{\partial x_{j} \partial x_{j^{3}}}+4 \frac{\partial f_{c, d}^{(1)}}{\partial x_{j}}-4 \frac{\partial f_{c, d+1}^{(1)}}{\partial x_{j}}+\frac{\partial^{2} f_{c, d}^{(0)}}{\partial x_{j}^{2}}+\frac{\partial^{2} f_{c, d+1}^{(0)}}{\partial x_{j}^{2}}\right] \\
+2\left(d\left(f_{c, d+1}^{(0)}-f_{c, d}^{(0)}\right)+f_{c, d+1}^{(0)}\right)-4 x_{j}^{3}\left[\frac{\partial f_{c, d}^{(0)}}{\partial x_{j^{3}}}-\frac{\partial f_{c, d+1}^{(0)}}{\partial x_{j^{3}}}+\frac{\partial f_{c, d}^{(0)}}{\partial x_{j}}+\frac{\partial f_{c, d+1}^{(0)}}{\partial x_{j}}\right] \\
\left.-x_{j}\left[\frac{\partial f_{c, d}^{(0)}}{\partial x_{j^{3}}}-\frac{\partial f_{c, d+1}^{(0)}}{\partial x_{j^{3}}}+\frac{\partial f_{c, d}^{(0)}}{\partial x_{j}}+\frac{\partial f_{c, d+1}^{(0)}}{\partial x_{j}}\right]\right)-x_{j^{3}}\left(-x_{j}\left[-8 d f_{c, d}^{(1)}+8 d f_{c, d+1}^{(1)}+3 \frac{\partial f_{c, d}^{(0)}}{\partial x_{j^{3}}}\right.\right. \\
-\frac{\left.\partial f_{c, d+1}^{(0)}+8 f_{c, d+1}^{(1)}+3 \frac{\partial f_{c, d}^{(0)}}{\partial x_{j}}+\frac{\partial f_{c, d+1}^{(0)}}{\partial x_{j}}\right]-4(2 d+3)\left(d f_{c, d}^{(0)}-(d+1) f_{c, d+1}^{(0)}\right)}{}
\end{array}
$$




$$
\begin{aligned}
& +x_{j}^{2}\left(\frac{\partial^{2} f_{c, d}^{(0)}}{\partial x_{j^{3}}^{2}}-\frac{\partial^{2} f_{c, d+1}^{(0)}}{\partial x_{j^{3}}^{2}}+4 \frac{\partial f_{c, d}^{(1)}}{\partial x_{j^{3}}}-4 \frac{\partial f_{c, d+1}^{(1)}}{\partial x_{j^{3}}}+2 \frac{\partial^{2} f_{c, d}^{(0)}}{\partial x_{j} \partial x_{j^{3}}}+2 \frac{\partial^{2} f_{c, d+1}^{(0)}}{\partial x_{j} \partial x_{j^{3}}}+8 f_{c, d}^{(0)}-8 f_{c, d+1}^{(0)}\right. \\
+ & \left.\left.4 \frac{\partial f_{c, d}^{(1)}}{\partial x_{j}}+4 \frac{\partial f_{c, d+1}^{(1)}}{\partial x_{j}}+\frac{\partial^{2} f_{c, d}^{(0)}}{\partial x_{j}^{2}}-\frac{\partial^{2} f_{c, d+1}^{(0)}}{\partial x_{j}^{2}}\right)+4 x_{j}^{3}\left(\frac{\partial f_{c, d}^{(0)}}{\partial x_{j^{3}}}+\frac{\partial f_{c, d+1}^{(0)}}{\partial x_{j^{3}}}+\frac{\partial f_{c, d}^{(0)}}{\partial x_{j}}-\frac{\partial f_{c, d+1}^{(0)}}{\partial x_{j}}\right)\right)=0
\end{aligned}
$$

Finally, the coefficient of the $O(1)$ term gives another long equation that we will again not quote.

Apart from the partial differential equations obtained above, we also need to require that the dilatation operator is hermittian. Recall that

$$
\left(\beta^{\dagger}\right)_{r_{1}, j, j^{3}}^{(n, m)}(a, b, c)=\beta_{r_{1}+c, j+b, j^{3}+c}^{(n, m)}(-a,-b,-c)
$$

Thus, we require

$$
\beta_{r_{1}, j, j^{3}}^{(n, m)}(c, q, 0)=\beta_{r_{1}, j+q, j^{3}}^{(n, m)}(-c,-q, 0)=\beta_{r_{1}, j+q, j^{3}}^{(n, m)}(c,-q, 0)
$$

which implies that

$$
\begin{aligned}
& m f_{c, a}^{(0)}\left(x_{j}, x_{j^{3}}\right)+\sqrt{m} f_{c, a}^{(1)}\left(x_{j}, x_{j^{3}}\right)+f_{c, a}^{(2)}\left(x_{j}, x_{j^{3}}\right)+\frac{1}{\sqrt{m}} f_{(c, a)}^{(3)}\left(x_{j}, x_{j^{3}}\right) \\
= & m f_{c,-a}^{(0)}\left(x_{j}+\frac{a}{\sqrt{m}}, x_{j^{3}}\right)+\sqrt{m} f_{c,-a}^{(1)}\left(x_{j}+\frac{a}{\sqrt{m}}, x_{j^{3}}\right)+f_{c,-a}^{(2)}\left(x_{j}+\frac{a}{\sqrt{m}}, x_{j^{3}}\right) \\
& +\frac{1}{\sqrt{m}} f_{(c,-a)}^{(3)}\left(x_{j}+\frac{a}{\sqrt{m}}, x_{j^{3}}\right) .
\end{aligned}
$$

Our goal now is to solve the equations given above for the leading order of the functions introduced. There are two equations we will use: (5.6) and (5.10). Introduce the functions

$$
F_{+} \equiv f_{c, d}^{(0)}+f_{c, d+1}^{(0)} \quad F_{-} \equiv f_{c, d}^{(0)}-f_{c, d+1}^{(0)}
$$

In terms of these functions (5.6) becomes

$$
2 x_{j^{3}}\left[d F_{-}+\frac{F_{-}-F_{+}}{2}\right]+x_{j}^{2}\left[\frac{\partial F_{+}}{\partial x_{j^{3}}}-\frac{\partial F_{-}}{\partial x_{j}}\right]+x_{j} x_{j^{3}}\left[\frac{\partial F_{+}}{\partial x_{j}}-\frac{\partial F_{-}}{\partial x_{j^{3}}}\right]=0
$$

and (5.10) becomes

$$
2 x_{j^{3}}\left[d F_{-}+\frac{F_{-}-F_{+}}{2}\right]+x_{j}^{2}\left[\frac{\partial F_{+}}{\partial x_{j^{3}}}+\frac{\partial F_{-}}{\partial x_{j}}\right]+x_{j} x_{j^{3}}\left[\frac{\partial F_{+}}{\partial x_{j}}+\frac{\partial F_{-}}{\partial x_{j^{3}}}\right]=0 .
$$

Suming these two equations we learn that

$$
x_{j} \frac{\partial F_{-}}{\partial x_{j}}+x_{j^{3}} \frac{\partial F_{-}}{\partial x_{j^{3}}}=0
$$

which implies that

$$
F_{-}=F_{-}(u) \quad u=\frac{x_{j^{3}}}{x_{j}}
$$


Note that this holds for any $d$. If we set $d=p$, since $F_{-}=f_{c, p}^{(0)}$ we learn that $f_{c, p}^{(0)}=f_{c, p}^{(0)}(u)$. If we set $d=p-1$, since $F_{-}=f_{c, p-1}^{(0)}-f_{c, p}^{(0)}$ depends only on $u$ and we already argued that $f_{c, p}^{(0)}$ depends only on $u$, we learn that $f_{c, p-1}^{(0)}=f_{c, p-1}^{(0)}(u)$. We can keep going in this way and consequently we have actually proved that

$$
f_{c d}^{(0)}=f_{c d}^{(0)}(u)
$$

for any $d$. This is a dramatic simplification - we had a collection of functions of two variables and now we have a collection of functions that depend only on one variable.

Now, again set $d=p$. In this case $F_{+}=F_{-}=F(u)$. We find that (5.6) becomes

$$
x_{j} \frac{\partial F}{\partial x_{j^{3}}}+x_{j^{3}} \frac{\partial F}{\partial x_{j}}=-2 \frac{x_{j^{3}}}{x_{j}} p F
$$

which has the general solution

$$
F=f_{c, p}^{(0)}=\kappa_{p}\left(1-u^{2}\right)^{p}=\kappa_{p}\left(1-\frac{x_{j^{3}}^{2}}{x_{j}^{2}}\right)^{p}
$$

where $\kappa_{p}$ is a constant. This has reproduced the correct answer for one loop when $p=1$ and has determined the leading order to an infinite number of higher loop dilatation operator coefficients.

Now, return to (5.16), and rewrite it using the new variable $y=1-u^{2}$ to obtain the simple form

$$
y \frac{d f_{c, d}^{(0)}}{d y}+y \frac{d f_{c, d+1}^{(0)}}{d y}=d f_{c, d}^{(0)}-(d+1) d f_{c, d+1}^{(0)} .
$$

If we now, set $d=p-1$ in (5.23) we can solve to obtain

$$
f_{c, p-1}^{(0)}=-2 p \kappa_{p} y^{p}+\kappa_{p-1} y^{p-1} .
$$

Next, set $d=p-2$ in (5.23) and again solve to obtain

$$
f_{c, p-2}^{(0)}=p(2 p-1) \kappa_{p} y^{p}-2(p-1) \kappa_{p-1} y^{p-1}+\kappa_{p-2} y^{p-2} .
$$

It is clear that we could continue with this process and determine all of the $f_{c, d}^{(0)}$. We have however determined all that we will need about the leading order. We will now show that we can determine the one loop answer and then return to the general $p$-loop analysis.

\subsection{One loop}

To determine the next to leading order, plug $d=1$ and the known leading order functions into (5.7) to obtain

$$
\frac{2}{x_{j^{3}}}\left(\frac{\partial f_{c, 1}^{(1)}}{\partial x_{j^{3}}}-\frac{\partial f_{c, 1}^{(1)}}{\partial x_{j}}\right) x_{j}^{4}\left(x_{j}-x_{j^{3}}\right)+4 f_{c, 1}^{(1)} x_{j}^{3}-\kappa_{1}\left(2 x_{j}-x_{j^{3}}\right)\left(x_{j}+x_{j^{3}}\right)=0,
$$


plug $d=0$ and the known leading order functions into (5.7) to obtain

$$
\begin{aligned}
& x_{j}^{3} x_{j^{3}}\left(x_{j}\left[-\frac{\partial f_{c, 0}^{(1)}}{\partial x_{j^{3}}}+\frac{\partial f_{c, 1}^{(1)}}{\partial x_{j^{3}}}+\frac{\partial f_{c, 0}^{(1)}}{\partial x_{j}}+\frac{\partial f_{c, 1}^{(1)}}{\partial x_{j}}\right]-2 f_{c, 1}^{(1)}\right) \\
+ & x_{j}^{5}\left(\frac{\partial f_{c, 0}^{(1)}}{\partial x_{j^{3}}}+\frac{\partial f_{c, 1}^{(1)}}{\partial x_{j^{3}}}-\frac{\partial f_{c, 0}^{(1)}}{\partial x_{j}}+\frac{\partial f_{c, 1}^{(1)}}{\partial x_{j}}\right)+\kappa_{1} x_{j} x_{j^{3}}^{2}+\kappa_{1} x_{j^{3}}^{3}=0,
\end{aligned}
$$

and finally, plug $d=-2$ and the known leading order functions into (5.7) to obtain

$$
x_{j}\left(\frac{\partial f_{c,-1}^{(1)}}{\partial x_{j^{3}}}+\frac{\partial f_{c,-1}^{(1)}}{\partial x_{j}}\right)\left(x_{j}+x_{j^{3}}\right)+2 f_{c,-1}^{(1)} x_{j^{3}}=0 .
$$

Next, plug $d=1$ and the known leading order functions into (5.11) to obtain

$$
-\frac{2}{x_{j^{3}}}\left(\frac{\partial f_{c, 1}^{(1)}}{\partial x_{j^{3}}}+\frac{\partial f_{c, 1}^{(1)}}{\partial x_{j}}\right) x_{j}^{4}\left(x_{j}+x_{j^{3}}\right)-\left(4 f_{c, 1}^{(1)} x_{j}^{3}+\kappa_{1}\left(-2 x_{j}^{2}+x_{j} x_{j^{3}}+x_{j^{3}}^{2}\right)\right)=0,
$$

plug $d=0$ and the known leading order functions into (5.11) to obtain

$$
\begin{gathered}
-x_{j}^{3} x_{j^{3}}\left(x_{j}\left[\frac{\partial f_{c, 0}^{(1)}}{\partial x_{j^{3}}}-\frac{\partial f_{c, 1}^{(1)}}{\partial x_{j^{3}}}+\frac{\partial f_{c, 0}^{(1)}}{\partial x_{j}}+\frac{\partial f_{c, 1}^{(1)}}{\partial x_{j}}\right]-2 f_{c, 1}^{(1)}\right) \\
-x_{j}^{5}\left(\frac{\partial f_{c, 0}^{(1)}}{\partial x_{j^{3}}}+\frac{\partial f_{c, 1}^{(1)}}{\partial x_{j^{3}}}+\frac{\partial f_{c, 0}^{(1)}}{\partial x_{j}}-\frac{\partial f_{c, 1}^{(1)}}{\partial x_{j}}\right)+\kappa_{1} x_{j} x_{j^{3}}^{2}-\kappa_{1} x_{j^{3}}^{3}=0,
\end{gathered}
$$

and finally, plug $d=-2$ and the known leading order functions into (5.11) to obtain

$$
-x_{j}\left(\frac{\partial f_{c,-1}^{(1)}}{\partial x_{j^{3}}}-\frac{\partial f_{c,-1}^{(1)}}{\partial x_{j}}\right)\left(x_{j}-x_{j^{3}}\right)-2 f_{c,-1}^{(1)} x_{j^{3}}=0 .
$$

We will now solve the above 6 partial differential equations simultaneously. To start, sum (5.26) and (5.29) which leads to

$$
4\left(x_{j} \frac{\partial f_{c, 1}^{(1)}}{\partial x_{j}}+x_{j^{3}} \frac{\partial f_{c, 1}^{(1)}}{\partial x_{j^{3}}}\right)+2 \kappa_{1} \frac{x_{j^{3}}^{2}}{x_{j}^{3}}=0 .
$$

The most general solution, regular at $x_{j^{3}}=0$ is

$$
f_{c, 1}^{(1)}=\frac{\kappa_{1}}{2} \frac{x_{j^{3}}^{2}}{x_{j}^{3}}+\sum_{n=0}^{\infty} c_{n} \frac{x_{j^{3}}^{n}}{x_{j}^{n}} .
$$

Inserting this solution into (5.26) we find

$$
\sum_{n} 2 c_{n} x_{j}^{3-n} x_{j^{3}}^{n-2}\left(n x_{j}^{2}-(n-2) x_{j^{3}}^{2}\right)=0 .
$$


Rearranging a little we find

$$
\sum_{m=-2}^{\infty} 2 c_{m+2}(m+2) x_{j}^{3-m} x_{j^{3}}^{m}-\sum_{n=0}^{\infty} 2 c_{n}(n-2) x_{j}^{3-n} x_{j^{3}}^{n}=0 .
$$

From the coefficient of $x_{j} x_{j^{3}}^{2}$ we have $4 c_{4}=0$. From the coefficient of $x_{j}^{-1-2 k} x_{j^{3}}^{4+2 k}$ we have $(6+2 k) c_{6+2 k}=(4+2 k) c_{4+2 k}$ which together implies $c_{2 k}=0$ for $k \geq 2$. From the coefficient of $x_{j}^{3}$ we have $2 c_{2}=-2 c_{0}$. This just shifts the constant $\kappa_{1}$ appearing in $f_{c, 1}^{(0)}$ by a term of $O\left(\frac{1}{\sqrt{m}}\right)$ and we may as well set it to zero. We shoud have expected this as we described in the last section, this is one of the symmetries that are present in our equations. By setting the coefficient of $x_{j}^{4} x_{j^{3}}^{-1}$ to zero we find $c_{1}=0$ and from the coefficient of $x_{j}^{4-2 k} x_{j^{3}}^{-1+2 k}$ we find $c_{2 k+1}=0$ for $k>1$. Putting everything together we only get a solution if all the coefficients $c_{n}=0$. Thus, we finally obtain

$$
f_{c, 1}^{(1)}=\frac{\kappa_{1}}{2} \frac{x_{j^{3}}^{2}}{x_{j}^{3}}
$$

which is indeed the correct answer.

Now, consider (5.28) and (5.31). From these two equations we can solve for $\frac{\partial f_{c,-1}^{(1)}}{\partial x_{j}}$ and for $\frac{\partial f_{c,-1}^{(1)}}{\partial x_{j}}$

$$
\frac{\partial f_{c,-1}^{(1)}}{\partial x_{j}}=-\frac{4 x_{j^{3}}^{2} f_{c,-1}^{(1)}}{x_{j}\left(x_{j}^{2}-x_{j^{3}}^{2}\right)}, \quad \frac{\partial f_{c,-1}^{(1)}}{\partial x_{j^{3}}}=\frac{4 x_{j^{3}} f_{c,-1}^{(1)}}{x_{j}^{2}-x_{j^{3}}^{2}} .
$$

These two equations are integrable - they give the same answer for $\frac{\partial^{2} f_{c, 1}^{(1)}}{\partial x_{j} \partial x_{j^{3}}}$. The only solution again corresponds to shifting $\kappa_{1}$, so that up to symmetry the most general solution is

$$
f_{c,-1}^{(1)}=0
$$

which is again the correct answer.

Finally, consider (5.27) and (5.30). After plugging in the solution we found for $f_{c, 1}^{(1)}$ we find

$$
2 x_{j}^{4}\left(\frac{\partial f_{c, 0}^{(1)}}{\partial x_{j^{3}}}-\frac{\partial f_{c, 0}^{(1)}}{\partial x_{j}}\right)+2 \kappa_{1} x_{j} x_{j^{3}}+3 \kappa_{1} x_{j^{3}}^{2}=0
$$

and

$$
2 x_{j}^{4}\left(\frac{\partial f_{c, 0}^{(1)}}{\partial x_{j^{3}}}+\frac{\partial f_{c, 0}^{(1)}}{\partial x_{j}}\right)+2 \kappa_{1} x_{j} x_{j^{3}}-3 \kappa_{1} x_{j^{3}}^{2}=0 .
$$

It is trivial to obtain the unique solution up to symmetry

$$
f_{c, 0}^{(1)}=-\frac{\kappa_{1}}{2} \frac{x_{j^{3}}^{2}}{x_{j}^{3}}
$$

which is again correct. This reproduces the complete leading correction at one loop. 
We can now check if our solution is hermittian which implies the following two conditions

$$
f_{c, a}^{(0)}\left(x_{j}, x_{j^{3}}\right)=f_{c,-a}^{(0)}\left(x_{j}, x_{j^{3}}\right)
$$

and

$$
f_{c, a}^{(1)}\left(x_{j}, x_{j^{3}}\right)=f_{c,-a}^{(1)}\left(x_{j}, x_{j^{3}}\right)+a \frac{\partial f_{c,-a}^{(0)}}{\partial x_{j}} .
$$

Recall that at one loop we have

$$
f_{c, \pm 1}^{(0)}=\frac{\kappa_{1}}{4}\left(1-\frac{x_{j^{3}}^{2}}{x_{j}^{2}}\right), \quad f_{c, 1}^{(1)}=\frac{\kappa_{1}}{2} \frac{x_{j^{3}}^{2}}{x_{j}^{3}}, \quad f_{c,-1}^{(1)}=0 .
$$

It is a non-trivial fact that

$$
f_{c, 1}^{(1)}\left(x_{j}, x_{j^{3}}\right)=f_{c,-1}^{(1)}\left(x_{j}, x_{j^{3}}\right)+\frac{\partial f_{c,-1}^{(0)}}{\partial x_{j}}
$$

so that our one loop solution is indeed Hermittian.

Finally, the next order is determined by the requirement that the $O(1)$ piece of (5.4) vanishes. Plugging in the solutions for $f^{(0)}, f^{(1)}$ as well as $d=1$, we find

$$
\begin{gathered}
16 x_{j}^{6}\left(\frac{\partial f_{c, 1}^{(2)}}{\partial x_{j^{3}}}-\frac{\partial f_{c, 1}^{(2)}}{\partial x_{j}}+x_{j^{3}} \kappa_{1}\right)+16 x_{j^{5}}^{5} x_{j^{3}}\left(-\frac{\partial f_{c, 1}^{(2)}}{\partial x_{j^{3}}}+\frac{\partial f_{c, 1}^{(2)}}{\partial x_{j}}+x_{j^{3}} \kappa_{1}\right) \\
-16 x_{j}^{4} x_{j^{3}}\left(-2 f_{c, 1}^{(2)}+x_{j^{3}}^{2} \kappa_{1}\right)-16 x_{j}^{7} \kappa_{1}-x_{j}^{3} \kappa_{1}+25 x_{j}^{2} x_{j^{3}} \kappa_{1}+25 x_{j} x_{j^{3}}^{2} \kappa_{1}-25 x_{j^{3}}^{3} \kappa_{1}=0
\end{gathered}
$$

and

$$
\begin{gathered}
-16 x_{j}^{6}\left(\frac{\partial f_{c, 1}^{(2)}}{\partial x_{j^{3}}}+\frac{\partial f_{c, 1}^{(2)}}{\partial x_{j}}+x_{j^{3}} \kappa_{1}\right)-16 x_{j}^{5} x_{j^{3}}\left(\frac{\partial f_{c, 1}^{(2)}}{\partial x_{j^{3}}}+\frac{\partial f_{c, 1}^{(2)}}{\partial x_{j}}-x_{j^{3}} \kappa_{1}\right) \\
+16 x_{j}^{4} x_{j^{3}}\left(-2 f_{c, 1}^{(2)}+x_{j^{3}}^{2} \kappa_{1}\right)-16 x_{j}^{7} \kappa_{1}-x_{j}^{3} \kappa_{1}-25 x_{j}^{2} x_{j^{3}} \kappa_{1}+25 x_{j} x_{j^{3}}^{2} \kappa_{1}+25 x_{j^{3}}^{3} \kappa_{1}=0 .
\end{gathered}
$$

Summing (5.45) and (5.46) we find

$$
x_{j} \frac{\partial f_{c, 1}^{(2)}}{\partial x_{j}}+x_{j^{3}} \frac{\partial f_{c, 1}^{(2)}}{\partial x_{j^{3}}}-x_{j^{3}}^{2} \kappa_{1}+x_{j}^{2} \kappa_{1}+\frac{\kappa_{1}}{16 x_{j}^{2}}-\frac{25 x_{j^{3}}^{2}}{16 x_{j}^{4}} \kappa_{1}=0 .
$$

The general solution to this equation is (again we have required that the solution is regular at $\left.x_{j^{3}}=0\right)$

$$
f_{c, 1}^{(2)}=\frac{x_{j^{3}}^{2}}{2} \kappa_{1}-\frac{x_{j}^{2}}{2} \kappa_{1}+\frac{\kappa_{1}}{32 x_{j}^{2}}-\frac{25 x_{j^{3}}^{2}}{32 x_{j}^{4}} \kappa_{1}+\sum_{n=0} a_{n} \frac{x_{j^{3}}^{n}}{x_{j}^{n}} .
$$


Plugging this into (5.45) we find

$$
\sum_{n=0}^{\infty} a_{n} x_{j}^{-n-3} x_{j^{3}}^{n-1}\left(n x_{j}^{2}-(n-2) x_{j^{3}}^{2}\right)=0 .
$$

The most general solution to this equation is $a_{0}=-a_{2}$ and $a_{n}=0$ for $n \neq 0,2$. You reach precisely the same conclusion if you use (5.46) instead of (5.45). Thus, our solution is

$$
f_{c, 1}^{(2)}=\frac{x_{j^{3}}^{2}}{2} \kappa_{1}-\frac{x_{j}^{2}}{2} \kappa_{1}+\frac{\kappa_{1}}{32 x_{j}^{2}}-\frac{25 x_{j^{3}}^{2}}{32 x_{j}^{4}} \kappa_{1}+k_{0}-k_{0} \frac{x_{j^{3}}^{2}}{x_{j}^{2}} .
$$

Setting $k_{0}=\frac{1}{2}$ and $\kappa_{1}=1$ we recover the answer from expanding the known dilatation operator coefficients.

Plugging in the solutions for $f^{(0)}, f^{(1)}$ as well as $d=0$ we find

$$
\begin{aligned}
& 16 x_{j}^{6}\left(\frac{\partial f_{c, 0}^{(2)}}{\partial x_{j^{3}}}+\frac{\partial f_{c, 1}^{(2)}}{\partial x_{j^{3}}}-\frac{\partial f_{c, 0}^{(2)}}{\partial x_{j}}+\frac{\partial f_{c, 1}^{(2)}}{\partial x_{j}}-x_{j^{3}} \kappa_{1}\right) \\
& -16 x_{j}^{5} x_{j^{3}}\left(\frac{\partial f_{c, 0}^{(2)}}{\partial x_{j^{3}}}-\frac{\partial f_{c, 1}^{(2)}}{\partial x_{j^{3}}}-\frac{\partial f_{c, 0}^{(2)}}{\partial x_{j}}-\frac{\partial f_{c, 1}^{(2)}}{\partial x_{j}}+x_{j^{3}} \kappa_{1}\right) \\
& +16 x_{j}^{4} x_{j^{3}}\left(x_{j^{3}}^{2} \kappa_{1}-2 f_{c, 1}^{(2)}\right)+16 x_{j}^{7} \kappa_{1}+x_{j}^{3} \kappa_{1}+11 x_{j}^{2} x_{j^{3}} \kappa_{1}-41 x_{j} x_{j^{3}}^{2} \kappa_{1}-43 x_{j^{3}}^{3} \kappa_{1}=0
\end{aligned}
$$

and

$$
\begin{array}{r}
-16 x_{j}^{6}\left(\frac{\partial f_{c, 0}^{(2)}}{\partial x_{j^{3}}}+\frac{\partial f_{c, 1}^{(2)}}{\partial x_{j^{3}}}+\frac{\partial f_{c, 0}^{(2)}}{\partial x_{j}}-\frac{\partial f_{c, 1}^{(2)}}{\partial x_{j}}-x_{j^{3}} \kappa_{1}\right) \\
-16 x_{j}^{5} x_{j^{3}}\left(\frac{\partial f_{c, 0}^{(2)}}{\partial x_{j^{3}}}-\frac{\partial f_{c, 1}^{(2)}}{\partial x_{j^{3}}}+\frac{\partial f_{c, 0}^{(2)}}{\partial x_{j}}+\frac{\partial f_{c, 1}^{(2)}}{\partial x_{j}}+x_{j^{3}} \kappa_{1}\right) \\
-16 x_{j}^{4} x_{j^{3}}\left(x_{j^{3}}^{2} \kappa_{1}-2 f_{c, 1}^{(2)}\right)+16 x_{j^{7}}^{7} \kappa_{1}+x_{j^{3}}^{3} \kappa_{1}-11 x_{j}^{2} x_{j^{3}} \kappa_{1}-41 x_{j} x_{j^{3}}^{2} \kappa_{1}+43 x_{j^{3}}^{3} \kappa_{1}=0 .
\end{array}
$$

Now, summing (5.51) and (5.52) we find

$$
-x_{j} \frac{\partial f_{c, 0}^{(2)}}{\partial x_{j}}-x_{j^{3}} \frac{\partial f_{c, 0}^{(2)}}{\partial x_{j^{3}}}+x_{j} \frac{\partial f_{c, 1}^{(2)}}{\partial x_{j}}+x_{j^{3}} \frac{\partial f_{c, 1}^{(2)}}{\partial x_{j^{3}}}-x_{j^{3}}^{2} \kappa_{1}+x_{j}^{2} \kappa_{1}+\frac{1}{16 x_{j}^{2}} \kappa_{1}-\frac{41 x_{j^{3}}^{2}}{16 x_{j}^{4}} \kappa_{1}=0 .
$$

Plugging in the solution for $f_{c, 1}^{(2)}$ that we constructed above, we find

$$
x_{j} \frac{\partial f_{c, 0}^{(2)}}{\partial x_{j}}+x_{j^{3}} \frac{\partial f_{c, 0}^{(2)}}{\partial x_{j^{3}}}+\frac{x_{j^{3}}^{2}}{x_{j}^{4}} \kappa_{1}=0
$$

which has the general solution

$$
f_{c, 0}^{(2)}=\frac{x_{j^{3}}^{2}}{2 x_{j}^{4}} \kappa_{1}+\sum_{n=0} a_{n} \frac{x_{j^{3}}^{n}}{x_{j}^{n}} .
$$


Inserting this solution into (5.51) we finally find

$$
f_{c, 0}^{(2)}=\frac{x_{j^{3}}^{2}}{2 x_{j}^{4}} \kappa_{1}+2 k_{0} \frac{x_{j^{3}}^{2}}{x_{j}^{2}}
$$

where $k_{0}$ is the same constant that appeared above.

Finally, plugging in the solutions for $f^{(0)}, f^{(1)}$ as well as $d=-2$ we find

$$
\begin{aligned}
& 16 x_{j}^{6}\left(\frac{\partial f_{c,-1}^{(2)}}{\partial x_{j^{3}}}+\frac{\partial f_{c,-1}^{(2)}}{\partial x_{j}}+x_{j^{3}} \kappa_{1}\right)-16 x_{j}^{5} x_{j^{3}}\left(-\frac{\partial f_{c,-1}^{(2)}}{\partial x_{j^{3}}}-\frac{\partial f_{c,-1}^{(2)}}{\partial x_{j}}+x_{j^{3}} \kappa_{1}\right) \\
- & 16 x_{j}^{4} x_{j^{3}}\left(-2 f_{c,-1}^{(2)}+x_{j^{3}}^{2} \kappa_{1}\right)+16 x_{j}^{7} \kappa_{1}+x_{j}^{3} \kappa_{1}+x_{j}^{2} x_{j^{3}} \kappa_{1}-x_{j} x_{j^{3}}^{2} \kappa_{1}-x_{j^{3}}^{3} \kappa_{1}=0
\end{aligned}
$$

and

$$
\begin{aligned}
& -16 x_{j}^{6}\left(\frac{\partial f_{c,-1}^{(2)}}{\partial x_{j^{3}}}-\frac{\partial f_{c,-1}^{(2)}}{\partial x_{j}}+x_{j^{3}} \kappa_{1}\right)-16 x_{j}^{5} x_{j^{3}}\left(-\frac{\partial f_{c,-1}^{(2)}}{\partial x_{j^{3}}}+\frac{\partial f_{c,-1}^{(2)}}{\partial x_{j}}+x_{j^{3}} \kappa_{1}\right) \\
& +16 x_{j}^{4} x_{j^{3}}\left(-2 f_{c,-1}^{(2)}+x_{j^{3}}^{2} \kappa_{1}\right)+16 x_{j}^{7} \kappa_{1}+x_{j}^{3} \kappa_{1}-x_{j}^{2} x_{j^{3}} \kappa_{1}-x_{j} x_{j^{3}}^{2} \kappa_{1}+x_{j^{3}}^{3} \kappa_{1}=0 .
\end{aligned}
$$

Summing (5.57) and (5.58) we find

$$
x_{j} \frac{\partial f_{c,-1}^{(2)}}{\partial x_{j}}+x_{j^{3}} \frac{\partial f_{c,-1}^{(2)}}{\partial x_{j^{3}}}-x_{j^{3}}^{2} \kappa_{1}+x_{j}^{2} \kappa_{1}+\frac{\kappa_{1}}{16 x_{j}^{2}}-\frac{x_{j^{3}}^{2}}{16 x_{j}^{4}} \kappa_{1}=0 .
$$

The general solution to this equation is (again we have required that the solution is regular at $\left.x_{j^{3}}=0\right)$

$$
f_{c,-1}^{(2)}=\frac{x_{j^{3}}^{2}}{2} \kappa_{1}-\frac{x_{j}^{2}}{2} \kappa_{1}+\frac{\kappa_{1}}{32 x_{j}^{2}}-\frac{x_{j^{3}}^{2}}{32 x_{j}^{4}} \kappa_{1}+\sum_{n=0} a_{n} \frac{x_{j^{3}}^{n}}{x_{j}^{n}} .
$$

Plugging this into (5.57) we find

$$
\sum_{n=0}^{\infty} a_{n} x_{j}^{-n-3} x_{j^{3}}^{n-1}\left(n x_{j}^{2}-(n-2) x_{j^{3}}^{2}\right)=0 .
$$

This is the equation we obtained above; the most general solution is $a_{0}=-a_{2}$ and $a_{n}=0$ for $n \neq 0,2$. Thus, our solution is

$$
f_{c,-1}^{(2)}=\frac{x_{j^{3}}^{2}}{2} \kappa_{1}-\frac{x_{j}^{2}}{2} \kappa_{1}+\frac{\kappa_{1}}{32 x_{j}^{2}}-\frac{x_{j^{3}}^{2}}{32 x_{j}^{4}} \kappa_{1}+\tilde{k}_{0}-\tilde{k}_{0} \frac{x_{j^{3}}^{2}}{x_{j}^{2}} .
$$

Setting $\tilde{k}_{0}=\frac{1}{2}$ we recover the answer from expanding the known dilatation operator coefficients. 
If we now study the $d=-1$ equation we can prove that $k_{0}=\tilde{k}_{0}$. Thus, in summary we have

$$
\begin{aligned}
f_{c, 1}^{(2)} & =\frac{x_{j^{3}}^{2}}{2} \kappa_{1}-\frac{x_{j}^{2}}{2} \kappa_{1}+\frac{\kappa_{1}}{32 x_{j}^{2}}-\frac{25 x_{j^{3}}^{2}}{32 x_{j}^{4}} \kappa_{1}+k_{0}-k_{0} \frac{x_{j^{3}}^{2}}{x_{j}^{2}}, \\
f_{c, 0}^{(2)} & =\frac{x_{j^{3}}^{2}}{2 x_{j}^{4}} \kappa_{1}+2 k_{0} \frac{x_{j^{3}}^{2}}{x_{j}^{2}}, \\
f_{c,-1}^{(2)} & =\frac{x_{j^{3}}^{2}}{2} \kappa_{1}-\frac{x_{j}^{2}}{2} \kappa_{1}+\frac{1}{32 x_{j}^{2}} \kappa_{1}-\frac{x_{j^{3}}^{2}}{32 x_{j}^{4}} \kappa_{1}+k_{0}-k_{0} \frac{x_{j^{3}}^{2}}{x_{j}^{2}} .
\end{aligned}
$$

Collecting the results we have found above, we have the three functions above as well as

$$
\begin{aligned}
& f_{c, 1}^{(0)}=\frac{\kappa_{1}}{4}\left(1-\frac{x_{j^{3}}^{2}}{x_{j}^{2}}\right), \quad f_{c, 0}^{(0)}=-\frac{\kappa_{1}}{2}\left(1-\frac{x_{j^{3}}^{2}}{x_{j}^{2}}\right), \quad f_{c,-1}^{(0)}=\frac{\kappa_{1}}{4}\left(1-\frac{x_{j^{3}}^{2}}{x_{j}^{2}}\right) \\
& f_{c, 1}^{(1)}=\frac{\kappa_{1}}{2} \frac{x_{j^{3}}^{2}}{x_{j}^{3}}, \quad f_{c, 0}^{(1)}=-\frac{\kappa_{1}}{2} \frac{x_{j^{3}}^{2}}{x_{j}^{3}}, \quad f_{c,-1}^{(1)}=0 .
\end{aligned}
$$

Requiring that the smallest eigenvalue of the one loop dilatation operator is zero determines $k_{0}=0$. Thus, up to an overall normalization which our argument can't determine, we have again reproduced (3.2).

\subsection{General discussion}

In this section we will extended our arguments to higher loops. More specifically, in the language of the discussion towards the end of section 4, our goal is to construct the most general solution up to symmetry. Recall that we have already determined (see (5.22) and (5.24) above)

$$
\begin{aligned}
f_{c, p}^{(0)} & =\kappa_{p}\left(1-\frac{x_{j^{3}}^{2}}{x_{j}^{2}}\right)^{p}, \\
f_{c, p-1}^{(0)} & =-2 p \kappa_{p}\left(1-\frac{x_{j^{3}}^{2}}{x_{j}^{2}}\right)^{p}+\kappa_{p-1}\left(1-\frac{x_{j^{3}}^{2}}{x_{j}^{2}}\right)^{p-1} .
\end{aligned}
$$

Plug $d=p$ and the known leading order functions into (5.7) to obtain

$$
\begin{gathered}
x_{j}\left(x_{j}-x_{j^{3}}\right)\left(x_{j}\left[\frac{\partial f_{c, p}^{(1)}}{\partial x_{j^{3}}}-\frac{\partial f_{c, p}^{(1)}}{\partial x_{j}}\right]\left(x_{j}-x_{j^{3}}\right)+2 f_{c, p}^{(1)} p x_{j^{3}}\right) \\
+\kappa_{p}\left(2(p-1) x_{j}^{4}+x_{j^{3}}^{2}\left(2(p-1) x_{j}^{2}+p(p+1)\right)-2 x_{j} x_{j^{3}}\left(2(p-1) x_{j}^{2}+p(p+1)\right)\right)\left(1-\frac{x_{j^{3}}^{2}}{x_{j}^{2}}\right)^{p}=0 .
\end{gathered}
$$

Plug $d=p$ and the known leading order functions into (5.11) to obtain

$$
\begin{aligned}
& \kappa_{p}\left(1-\frac{x_{j^{3}}^{2}}{x_{j}^{2}}\right)^{p}\left(2(p-1) x_{j}^{4}+4(p-1) x_{j}^{3} x_{j^{3}}+2(p-1) x_{j}^{2} x_{j^{3}}^{2}+2 p(p+1) x_{j} x_{j^{3}}+p(p+1) x_{j^{3}}^{2}\right) \\
&-x_{j}\left(x_{j}+x_{j^{3}}\right)\left(x_{j}\left[\frac{\partial f_{c, p}^{(1)}}{\partial x_{j^{3}}}+\frac{\partial f_{c, p}^{(1)}}{\partial x_{j}}\right]\left(x_{j}+x_{j^{3}}\right)+2 f_{c, p}^{(1)} p x_{j^{3}}\right)=0 .
\end{aligned}
$$


Summing these two we obtain

$$
x_{j} \frac{\partial f_{c, p}^{(1)}}{\partial x_{j}}+x_{j^{3}} \frac{\partial f_{c, p}^{(1)}}{\partial x_{j^{3}}}-\kappa_{p}\left(1-\frac{x_{j^{3}}^{2}}{x_{j}^{2}}\right)^{p-1}\left[2(p-1) x_{j}-p(p+1) \frac{x_{j^{3}}^{2}}{x_{j}^{3}}-2(p-1) \frac{x_{j^{3}}^{2}}{x_{j}}\right]=0 .
$$

The general solution to this equation, that is regular at $x_{j^{3}}=0$ is

$$
f_{c, p}^{(1)}=\kappa_{p}\left(1-\frac{x_{j^{3}}^{2}}{x_{j}^{2}}\right)^{p-1}\left[2(p-1) x_{j}+p(p+1) \frac{x_{j^{3}}^{2}}{x_{j}^{3}}-2(p-1) \frac{x_{j^{3}}^{2}}{x_{j}}\right]+\sum_{n=0}^{\infty} a_{n} \frac{x_{j^{3}}^{n}}{x_{j}^{n}} .
$$

Plugging this back into the first of our equations we find

$$
\sum_{n=0}^{\infty} a_{n} x_{j}^{-n} x_{j^{3}}^{n}\left[n\left(x_{j}-x_{j^{3}}\right)\left(x_{j}+x_{j^{3}}\right)+2 p x_{j^{3}}^{2}\right]=0 .
$$

The only solution is $a_{n}=0$ so that

$$
f_{c, p}^{(1)}=\kappa_{p}\left(1-\frac{x_{j^{3}}^{2}}{x_{j}^{2}}\right)^{p-1}\left[2(p-1) x_{j}+p(p+1) \frac{x_{j^{3}}^{2}}{x_{j}^{3}}-2(p-1) \frac{x_{j^{3}}^{2}}{x_{j}}\right] .
$$

Now, plug $d=p$ and the known leading order functions and $f_{c, p}^{(1)}$ into (5.7) to obtain

$$
\begin{array}{r}
x_{j}\left(x_{j}-x_{j^{3}}\right)^{2}\left(x_{j}+x_{j^{3}}\right)\left[x_{j}\left(\frac{\partial f_{c, p-1}^{(1)}}{\partial x_{j^{3}}}-\frac{\partial f_{c, p-1}^{(1)}}{\partial x_{j}}\right)\left(x_{j}-x_{j^{3}}\right)+2 f_{c, p-1}^{(1)}(p-1) x_{j^{3}}\right] \\
-\left(1-\frac{x_{j^{3}}^{2}}{x_{j}^{2}}\right)^{p}\left(x _ { j } ^ { 2 } x _ { j ^ { 3 } } ^ { 2 } \left(p\left(\kappa_{p}(6 p+3)+\kappa_{p-1}(-p)+\kappa_{p-1}+2(p-3) p-3\right)\right.\right. \\
\left.-2 x_{j}^{2}\left(\kappa_{p}\left(4 p^{2}-6 p+4\right)+\kappa_{p-1} p-2\left(\kappa_{p-1}+p\right)\right)\right) \\
+2 x_{j}^{6}\left(2 \kappa_{p}-(p-2)\left(\kappa_{p-1}-2 p\right)\right)+x_{j}^{3} x_{j^{3}}\left(4 x_{j}^{2}\left(\left(\kappa_{p}-1\right) p(2 p-3)+\kappa_{p-1}(p-2)\right)\right. \\
\left.-p\left(4 \kappa_{p} p+\kappa_{p}-2 \kappa_{p-1}(p-1)+4(p-1) p-1\right)\right) \\
+p x_{j} x_{j^{3}}^{3}\left(4 \kappa_{p} p^{2}-4\left(\kappa_{p}-1\right)(2 p-3) x_{j}^{2}+4 \kappa_{p} p+\kappa_{p}+4 p^{2}-4 p-1\right) \\
-\left(\kappa_{p}-1\right) p x_{j}^{4}-2 x_{j^{3}}^{4}\left(p\left(\kappa_{p}(p+1)(2 p+1)+(p-3) p-1\right)\right. \\
\left.\left.-2(p-1) x_{j}^{2}\left(\kappa_{p}(2 p-1)-p\right)\right)\right)=0
\end{array}
$$

and plug $d=p$ and the known leading order functions and $f_{c, p}^{(1)}$ into (5.11) to obtain

$$
\begin{array}{r}
-x_{j}\left(x_{j}-x_{j^{3}}\right)\left(x_{j}+x_{j^{3}}\right)^{2}\left[x_{j}\left(\frac{\partial f_{c, p-1}^{(1)}}{\partial x_{j^{3}}}+\frac{\partial f_{c, p-1}^{(1)}}{\partial x_{j}}\right)\left(x_{j}+x_{j^{3}}\right)+2 f_{c, p-1}^{(1)}(p-1) x_{j^{3}}\right] \\
\left(1-\frac{x_{j^{3}}^{2}}{x_{j}^{2}}\right)^{p}\left(x _ { j } ^ { 2 } x _ { j ^ { 3 } } ^ { 2 } \left(2 x _ { j } ^ { 2 } \left[\kappa_{p}\left(4 p^{2}-6 p+4\right)\right.\right.\right. \\
\left.\left.+\kappa_{p-1} p-2\left(\kappa_{p-1}+p\right)\right]-p\left[\kappa_{p}(6 p+3)+\kappa_{p-1}(-p)+\kappa_{p-1}+2(p-3) p-3\right]\right) \\
+2 x_{j}^{6}\left((p-2)\left(\kappa_{p-1}-2 p\right)-2 \kappa_{p}\right)+x_{j}^{3} x_{j^{3}}\left(4 x_{j}^{2}\left(\left(\kappa_{p}-1\right) p(2 p-3)+\kappa_{p-1}(p-2)\right)\right.
\end{array}
$$




$$
\begin{gathered}
\left.-p\left(4 \kappa_{p} p+\kappa_{p}-2 \kappa_{p-1}(p-1)+4(p-1) p-1\right)\right)+p x_{j} x_{j^{3}}^{3}\left(4 \kappa_{p} p^{2}\right. \\
\left.-4\left(\kappa_{p}-1\right)(2 p-3) x_{j}^{2}+4 \kappa_{p} p+\kappa_{p}+4 p^{2}-4 p-1\right)+\left(\kappa_{p}-1\right) p x_{j}^{4} \\
\left.+2 x_{j^{3}}^{4}\left(p\left(\kappa_{p}(p+1)(2 p+1)+(p-3) p-1\right)-2(p-1) x_{j}^{2}\left(\kappa_{p}(2 p-1)-p\right)\right)\right)=0 .
\end{gathered}
$$

Summing these two we obtain

$$
x_{j^{3}} \frac{\partial f_{c, p-1}^{(1)}}{\partial x_{j^{3}}}+x_{j} \frac{\partial f_{c, p-1}^{(1)}}{\partial x_{j}}+\left(1-\frac{x_{j^{3}}^{2}}{x_{j}^{2}}\right)^{p-2} F\left(x_{j}, x_{j^{3}}\right)=0,
$$

where

$$
\begin{aligned}
F\left(x_{j}, x_{j^{3}}\right)= & \frac{x_{j^{3}}^{2}}{x_{j}}\left(-8 \kappa_{p}+2 \kappa_{p-1} p-4 \kappa_{p-1}-8 p^{2} \kappa_{p}+16 p \kappa_{p}\right) \\
& +x_{j}\left(4 \kappa_{p}-2 \kappa_{p-1} p+4 \kappa_{p-1}+4 p^{2} \kappa_{p}-8 p \kappa_{p}\right)+2 p^{3} \kappa_{p} \frac{x_{j^{3}}^{4}}{x_{j}^{5}} \\
& +\frac{x_{j^{3}}^{2}}{x_{j}^{3}}\left(\kappa_{p-1} p^{2}-\kappa_{p-1} p-2 p^{3} \kappa_{p}\right)+\left(4 \kappa_{p}+4 p^{2} \kappa_{p}-8 p \kappa_{p}\right) \frac{x_{j^{3}}^{4}}{x_{j}^{3}} .
\end{aligned}
$$

The general solution to this equation, which is regular at $x_{j^{3}}=0$ is

$$
f_{c, p-1}^{(1)}=\left(1-\frac{x_{j^{3}}^{2}}{x_{j}^{2}}\right)^{p-2} G\left(x_{j}, x_{j^{3}}\right)+\sum_{n=0}^{\infty} a_{n} \frac{x_{j^{3}}^{n}}{x_{j}^{n}}
$$

where

$$
\begin{aligned}
G\left(x_{j}, x_{j^{3}}\right)= & -\frac{x_{j^{3}}^{2}}{x_{j}}\left(-8 \kappa_{p}+2 \kappa_{p-1} p-4 \kappa_{p-1}-8 p^{2} \kappa_{p}+16 p \kappa_{p}\right) \\
& -x_{j}\left(4 \kappa_{p}-2 \kappa_{p-1} p+4 \kappa_{p-1}+4 p^{2} \kappa_{p}-8 p \kappa_{p}\right)+2 p^{3} \kappa_{p} \frac{x_{j^{3}}^{4}}{x_{j}^{5}} \\
& +\frac{x_{j^{3}}^{2}}{x_{j}^{3}}\left(\kappa_{p-1} p^{2}-\kappa_{p-1} p-2 p^{3} \kappa_{p}\right)-\left(4 \kappa_{p}+4 p^{2} \kappa_{p}-8 p \kappa_{p}\right) \frac{x_{j^{3}}^{4}}{x_{j}^{3}} .
\end{aligned}
$$

Plugging this back into the first equation above we find

$$
x_{j}^{-n-1} x_{j^{3}}^{n-1}\left[a_{n} n x_{j}^{2}-a_{n} x_{j^{3}}^{2}(n-2 p+2)\right]=0
$$

which forces $a_{n}=0$.

Now, study the equation obtained by plugging $d=-p-1$ and the known leading order functions into (5.7) to obtain

$$
\begin{gathered}
x_{j}\left(x_{j}+x_{j^{3}}\right)\left(x_{j}\left[\frac{\partial f_{c,-p}^{(1)}}{\partial x_{j^{3}}}+\frac{\partial f_{c,-p}^{(1)}}{\partial x_{j}}\right]\left(x_{j}+x_{j^{3}}\right)+2 f_{c,-p}^{(1)} p x_{j^{3}}\right) \\
+\kappa_{p}(p-1)\left[x_{j^{3}}^{2}\left(p+2 x_{j}^{2}\right)+2 x_{j} x_{j^{3}}\left(p+2 x_{j}^{2}\right)+2 x_{j}^{4}\right]\left(1-\frac{x_{j^{3}}^{2}}{x_{j}^{2}}\right)^{p}=0 .
\end{gathered}
$$


Plug $d=p$ and the known leading order functions into (5.11) to obtain

$$
\begin{aligned}
& \kappa_{p}(p-1)\left(1-\frac{x_{j^{3}}^{2}}{x_{j}^{2}}\right)^{p}\left[x_{j^{3}}^{2}\left(p+2 x_{j}^{2}\right)-2 x_{j} x_{j^{3}}\left(p+2 x_{j}^{2}\right)+2 x_{j}^{4}\right] \\
& -x_{j}\left(x_{j}-x_{j^{3}}\right)\left(x_{j}\left[\frac{\partial f_{c,-p}^{(1)}}{\partial x_{j^{3}}}-\frac{\partial f_{c,-p}^{(1)}}{\partial x_{j}}\right]\left(x_{j}-x_{j^{3}}\right)+2 f_{c,-p}^{(1)} p x_{j^{3}}\right)=0 .
\end{aligned}
$$

Summing these two we obtain

$$
x_{j}^{3}\left(\frac{\partial f_{c,-p}^{(1)}}{\partial x_{j^{3}}} x_{j^{3}}+\frac{\partial f_{c,-p}^{(1)}}{\partial x_{j}} x_{j}\right)-\kappa_{p}(p-1)\left(1-\frac{x_{j^{3}}^{2}}{x_{j}^{2}}\right)^{p-2}\left[x_{j^{3}}^{2}\left(p+2 x_{j}^{2}\right)-2 x_{j}^{4}\right]=0 .
$$

The general solution to this equation, that is regular at $x_{j^{3}}=0$ is

$$
f_{c,-p}^{(1)}=\kappa_{p}(p-1)\left(1-\frac{x_{j^{3}}^{2}}{x_{j}^{2}}\right)^{p-1}\left(-p \frac{x_{j^{3}}^{2}}{x_{j}^{3}}+\frac{2 x_{j^{3}}^{2}}{x_{j}}-2 x_{j}\right)+\sum_{n=0}^{\infty} a_{n} \frac{x_{j^{3}}^{n}}{x_{j}^{n}} .
$$

Plugging this back into the first equation above we learn that $a_{n}=0$.

The only results we need from the above analysis are

$$
\begin{aligned}
f_{c, \pm p}^{(0)} & =\kappa_{p}\left(1-\frac{x_{j^{3}}^{2}}{x_{j}^{2}}\right)^{p}, \\
f_{c, p}^{(1)} & =\kappa_{p}\left(1-\frac{x_{j^{3}}^{2}}{x_{j}^{2}}\right)^{p-1}\left[2(p-1) x_{j}+p(p+1) \frac{x_{j^{3}}^{2}}{x_{j}^{3}}-2(p-1) \frac{x_{j^{3}}^{2}}{x_{j}}\right], \\
f_{c,-p}^{(1)} & =\kappa_{p}(p-1)\left(1-\frac{x_{j^{3}}^{2}}{x_{j}^{2}}\right)^{p-1}\left(-p \frac{x_{j^{3}}^{2}}{x_{j}^{3}}+\frac{2 x_{j^{3}}^{2}}{x_{j}}-2 x_{j}\right)+\sum_{n=0}^{\infty} a_{n} \frac{x_{j^{3}}^{n}}{x_{j}^{n}} .
\end{aligned}
$$

Now, computing

$$
f_{c, p}^{(1)}\left(x_{j}, x_{j^{3}}\right)-f_{c,-p}^{(1)}\left(x_{j}, x_{j^{3}}\right)-p \frac{\partial f_{c,-p}^{(0)}}{\partial x_{j}}=4 \kappa_{p}(p-1) x_{j}\left(1-\frac{x_{j^{3}}^{2}}{x_{j}^{2}}\right)^{p}
$$

we see that the only time that we get a Hermittian solution is when $p=1$. Thus we are forced to set $f_{c, \pm p}^{(0)}=f_{c, \pm p}^{(1)}=0$ which then implies that $f_{c, \pm p}^{(2)}=0$. We now apply the same argument to conclude that $f_{c, \pm p \mp 1}^{(0)}=f_{c, \pm p \mp 1}^{(1)}=f_{c, \pm p \mp 1}^{(2)}=0$ and keep going. Finally, when we get to $f_{c, \pm 1}^{(0)}, f_{c, \pm 1}^{(1)}, f_{c, \pm 1}^{(2)}$, we will find the one loop answer. This proves that the form of the piece of the dilatation operator that acts on the $Y$ fields is not corrected at any higher loop order.

\section{Acknowledgments}

This work is supported by the South African Research Chairs Initiative of the Department of Science and Technology and the National Research Foundation. Any opinion, findings 
and conclusions or recommendations expressed in this material are those of the authors and therefore the NRF and DST do not accept any liability with regard thereto. RdMK thanks Perimeter Institute for hospitality and for providing an excellent research environment. Research at Perimeter Institute is supported by the Government of Canada through Industry Canada and by the Province of Ontario through the Ministry of Economic Development \& Innovation.

\section{A The relation between $f_{c, d}^{(n, m)}\left(x_{j}, x_{j^{3}}\right)$ and $f_{c, d}^{(n-1, m+1)}\left(x_{j}, x_{j^{3}}\right)$}

In this appendix we derive a relation between $f_{c, d}^{(n, m)}\left(x_{j}, x_{j^{3}}\right)$ and $f_{c, d}^{(n-1, m+1)}\left(x_{j}, x_{j^{3}}\right)$ that is used extensively in section 5 . To make the discussion concrete we will study $f_{c, 0}^{(n, m)}\left(x_{j}, x_{j^{3}}\right)$ which is the continuum limit function corresponding to the following dilatation operator matrix element

$$
-\frac{1}{2}\left[m-\frac{(m+2)\left(j^{3}\right)^{2}}{j(j+1)}\right]
$$

This becomes the following function

$$
f_{c, 0}^{(n, m)}\left(x_{j}, x_{j^{3}}\right)=-\frac{1}{2}\left[m-\frac{(m+2)\left(\sqrt{m} x_{j^{3}}\right)^{2}}{\sqrt{m} x_{j}\left(\sqrt{m} x_{j}+1\right)}\right]
$$

We have the series expansion

$$
f_{c, 0}^{(n, m)}\left(x_{j}, x_{j^{3}}\right)=\sum_{q=0}^{\infty} m^{1-\frac{q}{2}} f_{c, 0}^{(m)}\left(x_{j}, x_{j^{3}}\right)
$$

When we replace $m \rightarrow m+1$, we do so without changing $j$ and $j^{3}$ - it is the expression (A.1) with $m \rightarrow m+1$ that solves the correct recursion relation. We must use the same definition of $x_{j}$ and $x_{j^{3}}$ for both $f_{c, 0}^{(n, m)}\left(x_{j}, x_{j^{3}}\right)$ and $f_{c, 0}^{(n-1, m+1)}\left(x_{j}, x_{j^{3}}\right)$, which implies that the new dilatation operator matrix element

$$
-\frac{1}{2}\left[m+1-\frac{(m+1+2)\left(j^{3}\right)^{2}}{j(j+1)}\right]
$$

leads to the following function

$$
f_{c, 0}^{(n-1, m+1)}\left(x_{j}, x_{j^{3}}\right)=-\frac{1}{2}\left[m+1-\frac{(m+1+2)\left(\sqrt{m} x_{j^{3}}\right)^{2}}{\sqrt{m} x_{j}\left(\sqrt{m} x_{j}+1\right)}\right] .
$$

We can get this function from $f_{c, 0}^{(n, m)}\left(x_{j}, x_{j^{3}}\right)$ by (i) shifting every $m \rightarrow m+1$ and then (ii) rescaling $x_{j} \rightarrow \sqrt{\frac{m}{m+1}} x_{j}$ and $x_{j^{3}} \rightarrow \sqrt{\frac{m}{m+1}} x_{j^{3}}$. In summary

$$
\begin{aligned}
f_{c, d}^{(n, m)}\left(x_{j}, x_{j^{3}}\right) & =\sum_{q=0}^{\infty} m^{1-\frac{q}{2}} f_{c, d}^{(m)}\left(x_{j}, x_{j^{3}}\right) \\
f_{c, d}^{(n-1, m+1)}\left(x_{j}, x_{j^{3}}\right) & =\sum_{q=0}^{\infty}(m+1)^{1-\frac{q}{2}} f_{c, d}^{(m)}\left(\sqrt{\frac{m}{m+1}} x_{j}, \sqrt{\frac{m}{m+1}} x_{j^{3}}\right) .
\end{aligned}
$$


Finally, note that

$$
\sqrt{\frac{m}{m+1}}=1-\frac{1}{2 m}+\frac{3}{8 m^{2}}+\ldots
$$

Open Access. This article is distributed under the terms of the Creative Commons Attribution License (CC-BY 4.0), which permits any use, distribution and reproduction in any medium, provided the original author(s) and source are credited.

\section{References}

[1] J.M. Maldacena, The Large- $N$ limit of superconformal field theories and supergravity, Adv. Theor. Math. Phys. 2 (1998) 231 [Int. J. Theor. Phys. 38 (1999) 1113] [hep-th/9711200] [INSPIRE].

[2] S.S. Gubser, I.R. Klebanov and A.M. Polyakov, Gauge theory correlators from noncritical string theory, Phys. Lett. B 428 (1998) 105 [hep-th/9802109] [INSPIRE].

[3] E. Witten, Anti-de Sitter space and holography, Adv. Theor. Math. Phys. 2 (1998) 253 [hep-th/9802150] [INSPIRE].

[4] J.A. Minahan and K. Zarembo, The Bethe ansatz for $N=4$ super Yang-Mills, JHEP 03 (2003) 013 [hep-th/0212208] [INSPIRE].

[5] N. Beisert, C. Kristjansen and M. Staudacher, The Dilatation operator of conformal $N=4$ super Yang-Mills theory, Nucl. Phys. B 664 (2003) 131 [hep-th/0303060] [INSPIRE].

[6] N. Beisert et al., Review of AdS/CFT Integrability: An Overview, Lett. Math. Phys. 99 (2012) 3 [arXiv:1012.3982] [INSPIRE].

[7] W. Carlson, R. de Mello Koch and H. Lin, Nonplanar Integrability, JHEP 03 (2011) 105 [arXiv: 1101.5404] [INSPIRE].

[8] R. de Mello Koch, M. Dessein, D. Giataganas and C. Mathwin, Giant Graviton Oscillators, JHEP 10 (2011) 009 [arXiv:1108.2761] [INSPIRE].

[9] R. de Mello Koch, G. Kemp and S. Smith, From Large-N Nonplanar Anomalous Dimensions to Open Spring Theory, Phys. Lett. B 711 (2012) 398 [arXiv:1111.1058] [InSPIRE].

[10] R. de Mello Koch and S. Ramgoolam, A double coset ansatz for integrability in AdS/CFT, JHEP 06 (2012) 083 [arXiv: 1204.2153] [INSPIRE].

[11] V. Balasubramanian, D. Berenstein, B. Feng and M.-x. Huang, D-branes in Yang-Mills theory and emergent gauge symmetry, JHEP 03 (2005) 006 [hep-th/0411205] [INSPIRE].

[12] R. de Mello Koch, J. Smolic and M. Smolic, Giant Gravitons - with Strings Attached (I), JHEP 06 (2007) 074 [hep-th/0701066] [INSPIRE].

[13] R. de Mello Koch, J. Smolic and M. Smolic, Giant Gravitons - with Strings Attached (II), JHEP 09 (2007) 049 [hep-th/0701067] [INSPIRE].

[14] D. Bekker, R. de Mello Koch and M. Stephanou, Giant Gravitons - with Strings Attached. III., JHEP 02 (2008) 029 [arXiv:0710.5372] [INSPIRE].

[15] R. Bhattacharyya, S. Collins and R. de Mello Koch, Exact Multi-Matrix Correlators, JHEP 03 (2008) 044 [arXiv: 0801.2061] [INSPIRE]. 
[16] V. Balasubramanian, M. Berkooz, A. Naqvi and M.J. Strassler, Giant gravitons in conformal field theory, JHEP 04 (2002) 034 [hep-th/0107119] [INSPIRE].

[17] S. Corley, A. Jevicki and S. Ramgoolam, Exact correlators of giant gravitons from dual $N=4$ SYM theory, Adv. Theor. Math. Phys. 5 (2002) 809 [hep-th/0111222] [INSPIRE].

[18] Y. Kimura and S. Ramgoolam, Branes, anti-branes and brauer algebras in gauge-gravity duality, JHEP 11 (2007) 078 [arXiv: 0709.2158] [INSPIRE].

[19] T.W. Brown, P.J. Heslop and S. Ramgoolam, Diagonal multi-matrix correlators and BPS operators in $N=4$ SYM, JHEP 02 (2008) 030 [arXiv:0711.0176] [INSPIRE].

[20] T.W. Brown, P.J. Heslop and S. Ramgoolam, Diagonal free field matrix correlators, global symmetries and giant gravitons, JHEP 04 (2009) 089 [arXiv:0806.1911] [INSPIRE].

[21] J. Pasukonis and S. Ramgoolam, From counting to construction of BPS states in $N=4$ SYM, JHEP 02 (2011) 078 [arXiv: 1010.1683] [INSPIRE].

[22] Y. Kimura and S. Ramgoolam, Enhanced symmetries of gauge theory and resolving the spectrum of local operators, Phys. Rev. D 78 (2008) 126003 [arXiv:0807.3696] [INSPIRE].

[23] Y. Kimura, Quarter BPS classified by Brauer algebra, JHEP 05 (2010) 103 [arXiv: 1002.2424] [INSPIRE].

[24] J. Pasukonis and S. Ramgoolam, Quantum states to brane geometries via fuzzy moduli spaces of giant gravitons, JHEP 04 (2012) 077 [arXiv: 1201.5588] [INSPIRE].

[25] Y. Kimura, Correlation functions and representation bases in free $N=4$ Super Yang-Mills, Nucl. Phys. B 865 (2012) 568 [arXiv:1206.4844] [INSPIRE].

[26] R. de Mello Koch, G. Kemp, B.A.E. Mohammed and S. Smith, Nonplanar integrability at two loops, JHEP 10 (2012) 144 [arXiv: 1206.0813] [INSPIRE].

[27] N. Beisert, The SU(2|3) dynamic spin chain, Nucl. Phys. B 682 (2004) 487 [hep-th/0310252] [INSPIRE].

[28] R. de Mello Koch, P. Diaz and N. Nokwara, Restricted Schur Polynomials for Fermions and integrability in the $\mathrm{SU}(2 \mid 3)$ sector, JHEP 03 (2013) 173 [arXiv:1212.5935] [INSPIRE].

[29] D. Berenstein, Giant gravitons: a collective coordinate approach, Phys. Rev. D 87 (2013) 126009 [arXiv:1301.3519] [InSPIRE].

[30] D. Berenstein and E. Dzienkowski, Open spin chains for giant gravitons and relativity, JHEP 08 (2013) 047 [arXiv:1305.2394] [INSPIRE].

[31] R. Bhattacharyya, R. de Mello Koch and M. Stephanou, Exact Multi-Restricted Schur Polynomial Correlators, JHEP 06 (2008) 101 [arXiv:0805.3025] [INSPIRE].

[32] V. De Comarmond, R. de Mello Koch and K. Jefferies, Surprisingly Simple Spectra, JHEP 02 (2011) 006 [arXiv: 1012.3884] [INSPIRE].

[33] V. Balasubramanian, M.-x. Huang, T.S. Levi and A. Naqvi, Open strings from $N=4$ super Yang-Mills, JHEP 08 (2002) 037 [hep-th/0204196] [INSPIRE].

[34] D. Berenstein, D.H. Correa and S.E. Vazquez, Quantizing open spin chains with variable length: An Example from giant gravitons, Phys. Rev. Lett. 95 (2005) 191601 [hep-th/0502172] [INSPIRE].

[35] D. Berenstein, D.H. Correa and S.E. Vazquez, A Study of open strings ending on giant gravitons, spin chains and integrability, JHEP 09 (2006) 065 [hep-th/0604123] [INSPIRE]. 